\title{
Meta-Symplectic Geometry of $3^{\text {rd }}$ Order Monge-Ampère Equations and their Characteristics ${ }^{\star}$
}

\author{
Gianni MANNO ${ }^{\dagger}$ and Giovanni MORENO ${ }^{\ddagger}$ \\ $\dagger$ Dipartimento di Scienze Matematiche "G.L. Lagrange", Politecnico di Torino, \\ Corso Duca degli Abruzzi 24, 10129 Torino, Italy \\ E-mail: giovanni.manno@polito.it \\ ¥ Institute of Mathematics, Polish Academy of Sciences, \\ ul. Śniadeckich 8, 00-656 Warsaw, Poland \\ E-mail: gmoreno@impan.pl
}

URL: https://www.impan.pl/en/sites/gmoreno/home

Received October 29, 2015, in final form March 16, 2016; Published online March 26, 2016

http://dx.doi.org/10.3842/SIGMA.2016.032

\begin{abstract}
This paper is a natural companion of [Alekseevsky D.V., Alonso Blanco R., Manno G., Pugliese F., Ann. Inst. Fourier (Grenoble) 62 (2012), 497-524, arXiv:1003.5177], generalising its perspectives and results to the context of third-order (2D) Monge-Ampère equations, by using the so-called "meta-symplectic structure" associated with the $8 \mathrm{D}$ prolongation $M^{(1)}$ of a $5 \mathrm{D}$ contact manifold $M$. We write down a geometric definition of a thirdorder Monge-Ampère equation in terms of a (class of) differential two-form on $M^{(1)}$. In particular, the equations corresponding to decomposable forms admit a simple description in terms of certain three-dimensional distributions, which are made from the characteristics of the original equations. We conclude the paper with a study of the intermediate integrals of these special Monge-Ampère equations, herewith called of Goursat type.
\end{abstract}

Key words: Monge-Ampère equations; prolongations of contact manifolds; characteristics of PDEs; distributions on manifolds; third-order PDEs

2010 Mathematics Subject Classification: 53D10; 35A30; 58A30; 14M15

\section{Introduction}

Classical Monge-Ampère equations (MAEs with one unknown function and two independent variables) constitute a distinguished class of scalar $2^{\text {nd }}$ order (non-linear) PDEs owing to a remarkable property: the totality of their characteristics, herewith called characteristic cone ${ }^{1}$, degenerates into the union of zero, one or two 2D planes (according to the elliptic, parabolic or hyperbolic character of the equation).

The primary motivation of this paper was to determine whether, and to what extent, such a phenomenon occurs also in the context of $3^{\text {rd }}$ order PDEs, where there can be found physically interesting analogues of the classical MAEs [1, 13, 14, 15, 28]. To the authors' best knowledge, such PDEs were defined by Boillat by using Lax's complete exceptionality [9, 10, 21]. We introduce now, in a friendly coordinate way, the notion of characteristic cone, whose intrinsic definition will be given later on (see (1.9) and (5.9)).

${ }^{\star}$ This paper is a contribution to the Special Issue on Analytical Mechanics and Differential Geometry in honour of Sergio Benenti. The full collection is available at http://www.emis.de/journals/SIGMA/Benenti.html

${ }^{1}$ The notion behind it is rather old, and may have appeared in other guises someplace else. 


\section{The main idea: studying PDEs using the geometry of their characteristics}

Let

$$
\mathcal{E}: F\left(x^{1}, x^{2}, u, p_{1}, p_{2}, \ldots, p_{i_{1} \cdots i_{l}}, \ldots\right)=0, \quad l \leq k,
$$

be a scalar $k^{\text {th }}$ order PDE with one unknown function $u=u\left(x^{1}, x^{2}\right)$ and two independent variables $\left(x^{1}, x^{2}\right)$. As usual (see [16] and, more recently, e.g., [18, 19] and references therein) in the geometric theory of PDEs, the variables $p_{i_{1} \cdots i_{l}}$ correspond to the partial derivatives $\frac{\partial^{l} u}{\partial x^{i_{1} \cdots \partial x^{i} l}}$, with the indices $i_{1} \leq i_{2} \leq \cdots \leq i_{l}$ ranging in $\{1,2\}$.

A Cauchy problem is obtained by complementing (1.1) with some initial conditions

$$
f\left(X^{1}(t), X^{2}(t)\right)=U(t), \quad \frac{\partial^{\ell} f}{\partial z^{\ell}}\left(X^{1}(t), X^{2}(t)\right)=Q_{\ell}(t), \quad \ell \leq k-1,
$$

where

$$
X_{1}(t), X_{2}(t), U(t), Q_{\ell}(t)
$$

are given functions, and $\frac{\partial}{\partial z}$ is the derivative along the normal direction ${ }^{2}$ of the curve $\left(X^{1}(t)\right.$, $\left.X^{2}(t)\right)$. A solution of the Cauchy problem (1.1), (1.2) is a function $u=f\left(x^{1}, x^{2}\right)$ which, together with its derivatives, satisfies both (1.1) and (1.2). Initial data (1.3) can be used to construct the curve

$$
\Phi(t)=\left(X^{1}(t), X^{2}(t), U(t), P_{1}(t), P_{2}(t), \ldots, P_{i_{1} \cdots i_{l}}(t), \ldots\right), \quad l \leq k-1,
$$

in the space with coordinates $\left(x^{1}, x^{2}, u, \ldots, p_{i_{1} \cdots i_{l}}, \ldots\right), l \leq k-1$, which we can then interpret as a Cauchy datum (see, e.g., [25] for a jet-theoretic treatment of the space of Cauchy data). If such curve is non-characteristic for equation (1.1), then, assuming $F$ real analytic, Cauchy problem (1.1), (1.2) admits a (locally) unique analytic solution. Hence, characteristic curves play a crucial role in the analysis of Cauchy problems. They can be defined as follows.

A curve (1.4) is characteristic for $\mathcal{E}$ at the point ${ }^{3} \bar{m}^{k-1} \in \mathcal{E}$, i.e., a point

$$
\bar{m}^{k-1}=\left(\bar{x}^{1}, \bar{x}^{2}, \bar{u}, \ldots, \bar{p}_{i_{1} \cdots i_{l}}, \ldots\right), \quad l \leq k,
$$

whose coordinates satisfy (1.1), if the tangent vector $\nu=\dot{\Phi}\left(t_{0}\right)$ at the point

$$
\bar{m}^{k-2}=\Phi\left(t_{0}\right)=\left(\bar{x}^{1}, \bar{x}^{2}, \bar{u}, \ldots, \bar{p}_{i_{1} \cdots i_{l}}, \ldots\right), \quad l \leq k-1,
$$

such that

$$
\left.\sum_{\ell_{1}+\ell_{2}=k}(-1)^{\ell_{1}} \frac{\partial F}{\left.\partial \underbrace{p_{1} \cdots 12 \cdots 2}_{\ell_{1}}\right|_{\ell_{2}}}\right|_{m^{k-1}}\left(\nu^{2}\right)^{\ell_{1}}\left(\nu^{1}\right)^{\ell_{2}}=0
$$

where

$$
\nu=\nu^{1}\left(\partial_{x^{1}}+\bar{p}_{1} \partial_{u}+\sum_{\substack{1 \leq i_{2} \leq \cdots \leq i_{h} \leq 2 \\ h \leq k}} \bar{p}_{1 i_{2} \cdots i_{h}} \partial_{p_{i_{2} \cdots i_{h}}}\right)+\nu^{2}\left(\partial_{x^{2}}+\bar{p}_{2} \partial_{u}+\sum_{\substack{1 \leq i_{2} \leq \cdots \leq i_{h} \leq 2 \\ h \leq k}} \bar{p}_{2 i_{2} \cdots i_{h}} \partial_{p_{i_{2} \cdots i_{h}}}\right) .
$$

\footnotetext{
${ }^{2}$ We adopted the same notation used in [29], where the reader may also find a gentle introduction to the theory of characteristics and singularities of non-linear PDEs.

${ }^{3}$ The choice of notation " $\bar{m}^{k-2}$ " and " $\bar{m}^{k-1}$ " will be motivated later on.
} 
From (1.7) one can associate with any point (1.5) of $\mathcal{E}$ a number $\leq k$ of directions (1.8) (the polynomial (1.7) might possess multiple and/or imaginary roots) in the space with coordinates $\left(x^{1}, x^{2}, u, \ldots, p_{i_{1} \cdots i_{l}}, \ldots\right), l \leq k-1$. So, if we keep the point (1.6) fixed and let the point (1.5) vary in $\mathcal{E}$, the aforementioned directions form, in general, a cone.

The set $\mathcal{V}_{\bar{m}^{k-2}}:=\left\{\right.$ directions $\nu$ as in (1.8) $\mid \exists \bar{m}^{k-1} \in \mathcal{E}$ such that $\left(\nu^{1}, \nu^{2}\right)$ satisfies $\left.(1.7)\right\}$ is what we call the characteristic cone of $\mathcal{E}$ at $\bar{m}^{k-2}$, and the union

$$
\mathcal{V}^{\mathcal{E}}:=\bigcup_{\bar{m}^{k-2}} \mathcal{V}_{\bar{m}^{k-2}}^{\mathcal{E}}
$$

is a geometric object naturally associated to $\mathcal{E}$ (see Section 5.2 later on).

In this paper, we basically study those PDEs that correspond to $3 \mathrm{D}$ distributions on the $8 \mathrm{D}$ first prolongation of a 5D contact manifold. Since the correspondence equation $\leftrightarrow$ distribution is essentially the same as in the case of $2^{\text {nd }}$ order MAEs, the PDEs we are interested in are precisely the $3^{\text {rd }}$ order analogues of the classical (i.e., second-order) bidimensional MAEs.

Our main motivation was the apparent lack of a systematic geometric analysis of $3^{\text {rd }}$ order MAEs, carried out in the same spirit as for classical MAEs. Much as in the geometric approach to classical MAEs it is convenient to exploit the contact/symplectic geometry underlying $2^{\text {nd }}$ order PDEs $[4,5,12,20,22,26]$, to deal with $3^{\text {rd }}$ order MAEs we shall make use of the prolongation of a contact manifold, equipped with its Levi form (see, e.g., [27, Section 2]), a structure known as meta-symplectic, quickly reviewed below. It is worth stressing that our main gadget, i.e., the correspondence between $\mathcal{E}$ and $\mathcal{V}^{\mathcal{E}}$, is just an adaptation of similar techniques traditionally found in other areas of modern mathematics ${ }^{4}$. In consequence, in this paper there coexist differential and algebraic geometric constructions, and the authors are aware that this may give a certain feeling of incompatibility. Section 4.5 below should help in this concern.

\section{Structure of the paper}

Section 2 reviews the classical notions needed for the formulation of the main Theorems 3.3, 3.4 and 3.5, which is given in Section 3, and the Sections 4, 5 introduce new ideas, namely the threefold orthogonality and its relationship with the characteristics variety, necessary to the proof of the main results, worked out in Section 6. In Section 7 we study the intermediate integrals of Goursat-type $3^{\text {rd }}$ order MAEs in terms of their characteristics.

\section{Notation and conventions}

All objects and morphisms herewith considered are assumed of class $C^{\infty}$, up to mild exceptions (see Section 4.5 below).

We use the symbol "P" in order to avoid too many repetitions of the sentence "up to a conformal factor". One-dimensional linear object may be identified with their generators. As a rule, if $P \rightarrow X$ is a bundle, and $x \in X$, we denote by $P_{x}$ the fibre of it at $x$. Symbol $\mathcal{D}^{\prime}$ denotes the derived distribution $\mathcal{D}+[\mathcal{D}, \mathcal{D}]$ of a distribution $\mathcal{D}$. Differential forms on a manifold $N$ (resp., distribution $\mathcal{D}$ ) are denoted by $\Lambda^{*} N$ (resp., $\Lambda^{*} \mathcal{D}^{*}$ ) and $f_{*}$ denotes the tangent map of $f$. If $T$ is a tensor on $N$, or a distribution, we sometimes skip the index " $n$ " in $T_{n}$, if it is clear from the context that $T$ has been evaluated in $n \in N$. The symmetric tensor product is denoted by $\odot$. The Einstein summation convention will be used, unless otherwise specified. Also, when a pair $j k$ (resp., a triple $i j k$ ) runs in a summation, such summation is performed over $1 \leq j \leq k \leq 2$ (resp., $1 \leq i \leq j \leq k \leq 2$ ), unless stated differently. Throughout the paper, by $M^{(0)}, \mathcal{C}^{0}$, and $m^{(0)}$ we shall always mean $M, \mathcal{C}$, and $m$, respectively.

\footnotetext{
${ }^{4}$ Bäcklund transformation, double fibration transform, Penrose transform, etc. (see also [24] on this concern).
} 
Remark 1.1. If there is any bundle structure, or a vertical distribution, in the surrounding manifold, the symbol $\mathcal{D}^{v}$ denotes the vertical part of the distribution $\mathcal{D}$.

\section{Preliminaries on (prolongations of) contact manifolds, and (meta)symplectic structures}

\subsection{Contact manifolds, their prolongations and PDEs}

Throughout this paper, $(M, \mathcal{C})$ will be a $5 \mathrm{D}$ contact manifold, i.e., $\mathcal{C}$ is a completely nonintegrable distribution of hyperplanes on $M$ locally described as $\mathcal{C}=\operatorname{ker} \theta$, where the 1 -form $\theta$ is determined up to a conformal factor and $\theta \wedge d \theta \wedge d \theta \neq 0$. The restriction $\left.d \theta\right|_{\mathcal{C}}$ defines on each $\mathcal{C}_{m}, m \in M$, a conformal symplectic structure: Lagrangian (i.e., maximally $d \theta$-isotropic) planes of $\mathcal{C}_{m}$ are tangent to maximal integral submanifolds of $\mathcal{C}$ and, as such, their dimension is 2 . We denote by $\mathcal{L}\left(\mathcal{C}_{m}\right)$ the Grassmannian of Lagrangian planes of $\mathcal{C}_{m}$ and by

$$
\pi: M^{(1)}:=\coprod_{m \in M} \mathcal{L}\left(\mathcal{C}_{m}\right) \rightarrow M
$$

the bundle of Lagrangian planes, also known as the $1^{\text {st }}$ prolongation of $M$. The key property of the manifold $M^{(1)}$ is that it is naturally endowed with a $5 \mathrm{D}$ distribution, defined by

$$
\mathcal{C}_{m^{1}}^{1}:=\left\{\nu \in T_{m^{1}} M^{(1)} \mid \pi_{*}(\nu) \in L_{m^{1}}\right\}
$$

where $L_{m^{1}} \equiv m^{1}$ is a point of $M^{(1)}$ considered as a Lagrangian plane in $\mathcal{C}_{m}$. Let us denote by $\theta^{(1)}$ the set of 1 -forms on $M^{(1)}$ vanishing on $\mathcal{C}^{1}$. Then, by definition, a Lagrangian plane of $M^{(1)}$ is a $2 \mathrm{D}$ subspace which is $\pi$-horizontal ${ }^{5}$ and such that all the forms belonging to the differential ideal generated by $\theta^{(1)}$, vanish on it. In analogy with $(2.1)$, we define the $2^{\text {nd }}$ prolongation $M^{(2)}$ of a contact manifold $(M, \mathcal{C})$ as the first prolongation of $M^{(1)}$, that is

$$
M^{(2)}=\left(M^{(1)}\right)^{(1)}:=\left\{\text { Lagrangian planes of } M^{(1)}\right\} \text {. }
$$

Projection (2.1) is the beginning of a tower of natural bundles $M^{(2)} \stackrel{\pi_{2,1}}{\longrightarrow} M^{(1)} \stackrel{\pi}{\longrightarrow} M$, which, for the present purposes, will be exploited only up to its $2^{\text {nd }}$ term. It is well known that $\pi_{2,1}$ is an affine bundle (see, e.g., [17]).

The tautological bundle $L \rightarrow M^{(i)}$ is defined by requiring that the fibre $L_{m^{i}}$ is $m^{i}$ itself, understood as a $2 \mathrm{D}$ subspace of $\mathcal{C}^{i-1}$, with $i=1,2$. We keep the same symbol $L$ for both the tautological bundles over $M^{(1)}$ and $M^{(2)}$, since it will be clear from the context which is which.

A generic point of $M$ (resp., $M^{(1)}, M^{(2)}$ ) is denoted by $m$ (resp., $m^{1}, m^{2}$ ). As a rule, when both $m^{1}$ and $m^{2}$ (resp. $m$ and $m^{1}$ ) appear in the same context, the former is always the $\pi_{2,1}$-image (resp., the $\pi$-image) of the latter.

By a $k^{\text {th }}$ order PDE we always mean a sub-bundle $\mathcal{E} \subseteq M^{(k-1)}$ of codimension one whose fibre $\mathcal{E}_{m^{k-2}}$ at $m^{k-2}$ is henceforth assumed, without loss of generality, to be connected (see (4.26) below). Recalling that $\pi_{2,1}$ is an affine bundle, we say that an equation $\mathcal{E}$ is quasi-linear at a point $m^{1} \in M^{(1)}$ if the fibre $\mathcal{E}_{m^{1}}$ is an affine subspace of $\pi_{2,1}^{-1}\left(m^{1}\right)$, otherwise we say that it is non-linear at $m^{1}$. We retain the same symbol $L$ for the tautological bundle $\left.L\right|_{\mathcal{E}} \longrightarrow \mathcal{E}$ restricted to $\mathcal{E}$.

\footnotetext{
${ }^{5}$ If horizontality is dropped in $(2.2)$, one augments $M^{(2)}$ with the so-called "singular" integral elements of $\mathcal{C}^{1}$ which, in the case of PDEs, formalise the notion of "singular solutions", originally introduced in [23] (see also the review paper [29] and references therein).
} 


\subsection{The meta-symplectic structure on $\mathcal{C}^{1}$}

The (local) conformal symplectic structure $\varpi=\left.d \theta\right|_{\mathcal{C}}$ admits a "global" analog, namely

$$
\varpi_{\text {glob }}: \mathcal{C} \wedge \mathcal{C} \longrightarrow \frac{\mathcal{C}^{\prime}}{\mathcal{C}}=\frac{T M}{\mathcal{C}}
$$

Indeed, since $\frac{T M}{\mathcal{C}}$ is rank-one, $\varpi_{\text {glob }}$ locally identifies with $\varpi$.

One of the main gadgets of our analysis is the co-restriction to $\left(\mathcal{C}^{1}\right)^{\prime}$ of the Levi form of $\mathcal{C}^{1}$, firstly investigated by V. Lychagin [19] as a "twisted" analog of the symplectic form (2.3) for the prolonged contact distribution $\mathcal{C}^{1}$ and called, for this reason, meta-symplectic:

$$
\Omega: \mathcal{C}^{1} \wedge \mathcal{C}^{1} \longrightarrow \frac{\left(\mathcal{C}^{1}\right)^{\prime}}{\mathcal{C}^{1}}
$$

Notice that, unlike (2.3), the form (2.4) takes its values into a rank-two bundle so that, even locally, it cannot be regarded as a 2-form in the standard sense. Nevertheless, it can be used for defining Lagrangian subspaces: indeed, a $\pi_{2,1}$-horizontal $2 \mathrm{D}$ subspace of $\mathcal{C}_{m^{1}}^{1}$ is Lagrangian if it is $\Omega$-isotropic.

\section{Description of the main results}

In the case of a $3^{\text {rd }}$ order $\operatorname{PDE} \mathcal{E}$, polynomial (1.7) always admits a linear factor, so that the corresponding characteristic cone $\mathcal{V}^{\mathcal{E}}$, always contains a linear irreducible component $\mathcal{V}_{\mathrm{I}}^{\mathcal{E}}$ (we stress that in the fully decomposable case, all the three irreducible components are linear, and it suffices to choose one).

Definition 3.1. If $\omega \in \Lambda^{2} M^{(1)}$ is a 2-form, then the hypersurface

$$
\mathcal{E}_{\omega}:=\left\{m^{2} \in M^{(2)}|\omega|_{L_{m^{2}}}=0\right\}
$$

in $M^{(2)}$ is called the Boillat-type $3^{\text {rd }}$ order MAE (associated to $\omega$ ).

The first result of this paper is concerned with the characteristic cone $\mathcal{V}^{\mathcal{E}_{\omega}}$. Namely, we show that $\mathcal{V}^{\mathcal{E}_{\omega}}$ can be used to recover the equation itself, since there is an obvious way to "invert" the construction of the characteristic cone (1.9) out of a $\operatorname{PDE} \mathcal{E}$ : take any sub-bundle $\mathcal{V} \subseteq \mathbb{P} \mathcal{C}^{1}$ and associate with it the following subset

$$
\mathcal{E}_{\mathcal{V}}:=\left\{m^{2} \in M^{(2)} \mid \exists H \in \mathcal{V}: L_{m^{2}} \supset H\right\} \subseteq M^{(2)} .
$$

Now, if $\mathcal{V}$ is regular enough, the corresponding $\mathcal{E}_{\mathcal{V}}$ turns out to be a genuine $3^{\text {rd }}$ order PDE, and examples of "regular enough" sub-bundles are provided by characteristic cones of $3^{\text {rd }}$ order PDEs themselves. In particular, it always holds the inclusion $\mathcal{E}_{\mathcal{V}^{\mathcal{E}}} \supseteq \mathcal{E}$.

Definition 3.2. We say that the equation $\mathcal{E}$ is recoverable (from its characteristics) if

$$
\mathcal{E}=\mathcal{E}_{\mathcal{V}^{\mathcal{E}}}
$$

Theorem 3.3. Any $3^{\text {rd }}$ order $M A E$ is recoverable from its characteristics.

The simplest examples of equations (3.2) are obtained when $\mathcal{V}=\mathbb{P} \mathcal{D}$, where $\mathcal{D} \subset \mathcal{C}^{1}$ is a 3D sub-distribution:

$$
\mathcal{E}_{\mathcal{D}}:=\mathcal{E}_{\mathbb{P} \mathcal{D}}=\left\{m^{2} \in M^{(2)} \mid L_{m^{2}} \cap \mathcal{D}_{m^{1}} \neq 0\right\},
$$


with $m^{2}$ projecting onto $m^{1}$. Equations (3.4) are herewith dubbed Goursat-type $3^{\text {rd }}$ order MAEs. Observe that the equations (3.4) form the sub-class of the equations (3.1) which are determined by 2 -forms which are decomposable modulo the differential ideal generated by contact forms (see the beginning of Section 6.3). The second result of this paper allows to locally characterizes Goursat-type $3^{\text {rd }}$ order MAEs in terms of their characteristic cone.

Theorem 3.4. Let $\mathcal{E}$ be a $3^{\text {rd }}$ order PDE. Then $\mathcal{E}$ is locally of the form (3.4), if and only if its characteristic cone $\mathcal{V}^{\mathcal{E}}$ contains an irreducible component $\mathcal{V}_{\mathrm{I}}^{\mathcal{E}}$ which is a $2 D$ linear projective sub-bundle.

Theorem 3.4 implies that $\mathcal{E} \subseteq M^{(2)}$ is a Goursat-type $3^{\text {rd }}$ order MAE if and only if its characteristic cone decomposes as

$$
\mathcal{V}^{\mathcal{E}}=\mathbb{P} \mathcal{D}_{1} \cup \mathcal{V}_{\mathrm{II}}^{\mathcal{E}}
$$

where $\mathcal{D}_{1} \subseteq \mathcal{C}^{1}$ is a $3 \mathrm{D}$ sub-distribution and $\mathcal{V}_{\text {II }}^{\mathcal{E}}$ encompasses all the remaining irreducible components of $\mathcal{V}^{\mathcal{E}}$. Surprisingly enough, if (3.5) holds, then all the information about $\mathcal{E}$ is encapsulated in $\mathcal{D}_{1}$. For instance, if $\mathcal{V}_{\text {II }}^{\mathcal{E}}$ is in its turn reducible, then its components $\mathbb{P} \mathcal{D}_{2}$ and $\mathbb{P D}_{3}$ are linear as well and can be unambiguously characterized by $\mathbb{P} \mathcal{D}_{1}$ through the formula

$$
\Omega\left(h_{1}, \mathcal{D}_{i}^{v}\right)=\Omega\left(h_{i}, \mathcal{D}_{1}^{v}\right)=1 \mathrm{D} \text { space, }
$$

where $h_{i}$ is a non-zero horizontal vector in $\mathcal{D}_{i}, i=1,2,3$ (for the definition of $\mathcal{D}^{v}$ see Remark 1.1).

Observe that, if a Goursat-type MAE is non-linear in a point $m^{1} \in M^{(1)}$, then it is non-linear in a neighbourhood of $m^{1}$; on the contrary, if it is linear in $m^{1}$, then a neighbourhood of $m^{1}$ where it is quasi-linear may not exist (see (4.25)). On account of this, we give the following Theorem 3.5, that finalises our characterisation of Goursat-type $3^{\text {rd }}$ order MAEs through their characteristics.

Theorem 3.5. Let $\mathcal{E}=\mathcal{E}_{\mathbb{P} \mathcal{D}_{1}}$, where $\mathcal{D}_{1} \subset \mathcal{C}$ is a 3-dimensional distribution, be a Goursat-type $M A E$. Let $m^{1} \in M^{(1)}$. Then $\mathcal{E}=\mathcal{E}_{\mathbb{P} \mathcal{D}_{1} \cup \mathcal{V}_{\mathrm{II}}^{\mathcal{E}}}$ and

1) $\mathcal{E}$ is quasi-linear at $m^{1}$ if and only if $\operatorname{dim}\left(\mathcal{D}_{1}^{v}\right)_{m^{1}}=2$ : in this case, $\left(\mathcal{V}_{\mathrm{II}}^{\mathcal{E}}\right)_{m^{1}}$ is either empty or equal to $\mathbb{P}\left(\mathcal{D}_{2}\right)_{m^{1}} \cup \mathbb{P}\left(\mathcal{D}_{3}\right)_{m^{1}}$, where $\left(\mathcal{D}_{i}\right)_{m^{1}}$ is unambiguously defined by $(3.6)$ and $\mathcal{E}_{\left(\mathcal{D}_{i}\right)_{m} 1}=\mathcal{E}_{\left(\mathcal{D}_{1}\right)_{m} 1}, i=2,3$;

2) $\mathcal{E}$ is non-linear at $m^{1}$ if and only if $\operatorname{dim}\left(\mathcal{D}_{1}^{v}\right)_{m^{1}}=1$ : in this case, if $\left(\mathcal{V}_{\mathrm{II}}^{\mathcal{E}}\right)_{m^{1}}$ is not empty, then it cannot contain any linear irreducible component and $\mathcal{E}_{m^{1}}=\mathcal{E}_{\left(\mathcal{V}_{\mathrm{II}}^{\mathcal{E}}\right)_{m}^{1}}$;

3) if $\mathcal{E}_{m^{1}}=\mathcal{E}_{\left(\mathcal{D}_{2}\right)_{m^{1}}}$ for some $3 D$ subspace $\left(\mathcal{D}_{2}\right)_{m^{1}} \subset \mathcal{C}_{m^{1}}^{1}$, then either $\left(\mathcal{D}_{1}\right)_{m^{1}}=\left(\mathcal{D}_{2}\right)_{m^{1}}$ or $\left(\mathcal{D}_{1}\right)_{m^{1}}$ and $\left(\mathcal{D}_{2}\right)_{m^{1}}$ are "orthogonal" in the sense of (3.6).

As a main byproduct of Theorem 3.5 we shall obtain a method for finding intermediate integrals, discussed in Section 7. We conclude this section with a local description of the main objects introduced so far.

\subsection{Local coordinate description of the main objects}

Let $\left(x^{i}, u, p_{i}\right)$ be contact coordinates on $M, i=1,2$. Then $\theta=d u-p_{i} d x^{i}$, and

$$
\mathcal{C}=\left\langle D_{1}, D_{2}, \partial_{p_{1}}, \partial_{p_{2}}\right\rangle,
$$

where $D_{i}$ is the total derivative with respect to $x^{i}$, truncated to the $0^{\text {th }}$ order. The aboveintroduced system $\left(x^{i}, u, p_{i}\right)$ induces coordinates

$$
\left(x^{i}, u, p_{i}, p_{i j}=p_{j i}, 1 \leq i, j \leq 2\right)
$$


on $M^{(1)}$ as follows: a point $m^{1} \equiv L_{m^{1}} \in M^{(1)}$ has coordinates (3.8) iff $m=\pi\left(m^{1}\right)=\left(x^{i}, u, p_{i}\right)$ and the corresponding Lagrangian plane $L_{m^{1}}$ is given by

$$
L_{m^{1}}=\left\langle D_{i}+p_{i j} \partial_{p_{j}} \mid i \in\{1,2\}\right\rangle \subset \mathcal{C}_{m}
$$

Similarly,

$$
\mathcal{C}^{1}=\left\langle D_{1}, D_{2}, \partial_{p_{11}}, \partial_{p_{12}}, \partial_{p_{22}}\right\rangle,
$$

where now the $D_{i}$ 's stand for the total derivatives truncated to the $1^{\text {st }}$ order and a point $m^{2} \in M^{(2)}$ has coordinates $\left(x^{i}, u, p_{i}, p_{i j}=p_{j i}, p_{i j k}=p_{i k j}=p_{j i k}=p_{j k i}=p_{k i j}=p_{k j i}\right)$ if the corresponding Lagrangian plane is given by

$$
L_{m^{2}}=\left\langle D_{i}+p_{i j k} \partial_{p_{j k}} \mid i \in\{1,2\}\right\rangle \subseteq \mathcal{C}_{m^{1}}^{1},
$$

where the vector fields $D_{i}$ and $\partial_{p_{i j}}$ are tacitly assumed to be evaluated at $m^{1}$.

Remark 3.6. We always use the symbol $D_{i}$ for the truncated total derivative with respect to $x^{i}$, $i=1,2$, the order of truncation depending on the context. For instance, the order of truncation is 0 in (3.7) and it is 1 in (3.10) and (3.11). It is convenient to set

$$
\xi_{i}:=\left.D_{i}\right|_{m^{k}}, \quad k=1,2,
$$

where the total derivatives appearing in $(3.12)$ are truncated to the $(k-1)^{\text {st }}$ order. Indeed, both (3.9) and (3.11) simplify as

$$
L_{m^{k}}=\left\langle\xi_{1}, \xi_{2}\right\rangle, \quad k=1,2,
$$

and their dual as $L_{m^{k}}^{*}=\left\langle d x^{1}, d x^{2}\right\rangle$, respectively.

Using the local coordinates $(3.10)$, it is easy to realize that $\left(\mathcal{C}^{1}\right)^{\prime}$ is spanned by $\mathcal{C}^{1}$ and $\left\langle\partial_{p_{1}}, \partial_{p_{2}}\right\rangle$, so that the quotient $\frac{\left(\mathcal{C}^{1}\right)^{\prime}}{\mathcal{C}^{1}}$ identifies with the latter, and (2.4) reads

$$
\Omega=d p_{i j} \wedge d x^{i} \otimes \partial_{p_{j}} .
$$

As a vector-valued differential 2 -form, $\Omega$ can be identified with the pair $\Omega \equiv\left(-d \theta_{1},-d \theta_{2}\right)$ where $\theta_{i}=d p_{i}-p_{i j} d x^{j}$

\subsubsection{Boillat and Goursat $3^{\text {rd }}$ order MAEs}

We conclude with a local coordinate description of Boillat (3.1) and Goursat (3.4) equations $\mathcal{E}=\{F=0\}$. Locally, the former is given by

$$
F=\operatorname{det}\left(\begin{array}{ccc}
p_{111} & p_{112} & p_{122} \\
p_{112} & p_{122} & p_{222} \\
& \boldsymbol{A} &
\end{array}\right)+\boldsymbol{B} \cdot\left(p_{111}, p_{112}, p_{122}, p_{222}\right)^{T}+C
$$

where $\boldsymbol{A}$ (resp., $\boldsymbol{B}, C$ ) is an $\mathbb{R}^{3}$-(resp., $\mathbb{R}^{4}$-, $\mathbb{R}$-) valued smooth function on $M^{(1)}$, and the latter is either given by

$$
F=\operatorname{det}\left(\begin{array}{ccc}
p_{111}-f_{111} & p_{112}-f_{112} & p_{122}-f_{122} \\
p_{112}-f_{211} & p_{122}-f_{212} & p_{222}-f_{222} \\
\boldsymbol{A} &
\end{array}\right),
$$

where $\boldsymbol{A}$ is as in (3.15) and $f_{i j k} \in C^{\infty}\left(M^{(1)}\right)$, or it is quasi-linear. 


\section{Vertical geometry of (prolongations of) contact manifolds and their characteristics}

The departing point of our analysis of $3^{\text {rd }}$ order PDEs is to identify them with sub-bundles of the $1^{\text {st }}$ prolongation $M^{(2)}=\left(M^{(1)}\right)^{(1)}$ of $M^{(1)}$. Hence, a key role will be played by their vertical geometry, i.e., the $1^{\text {st }}$ order approximation of their bundle structure, and, in particular, by the so-called rank-one vectors, which are in turn linked to the notion of characteristics. In order to introduce these concepts, we begin with the vertical geometry of the surrounding bundle, i.e., $M^{(2)}$ itself.

\subsection{Vertical geometry of $M^{(k)}$ and three-fold orthogonality in $M^{(1)}$}

For $k \in\{1,2\}$, we define the vertical bundle over $M^{(k)}$ as follows

$$
V M^{(k)}:=\coprod_{m^{k} \in M^{(k)}} T_{m^{k}} M_{m^{k-1}}^{(k)} .
$$

As regard to the contact manifold $M$, i.e., the case $k=0$, it does not possess a naturally defined vertical bundle: we replace it by the following bundle on $M^{(1)}$ :

$$
V M:=\coprod_{m^{1} \in M^{(1)}} \frac{\mathcal{C}_{\pi\left(m^{1}\right)}}{L_{m^{1}}} .
$$

Lemma 4.1. It holds the following canonical isomorphism:

$$
V M^{(k)} \simeq S^{k+1} L^{*}, \quad k \geq 0 .
$$

Proof. It is a generalisation of the proof of the jet-theoretic version of the statement (see, e.g., [6, Theorem 3.2]).

Directly from the definition (4.1) of $V M$ and the isomorphism (4.2) for $k=0$ one obtains

$$
\frac{\mathcal{C}_{\pi\left(m^{1}\right)}}{L_{m^{1}}} \cong L_{m^{1}}^{*}
$$

Example 4.2. Of particular importance will be the vertical vectors on $M^{(2)}$ which correspond to the perfect cubes of covectors on $L$ via the fundamental isomorphism (4.2). For instance, if

$$
\alpha:=\alpha_{i} d x^{i} \in L_{m^{2}}^{*}
$$

(see Remark 3.6), then the corresponding vertical vector is

$$
\left.S^{3} L_{m^{2}}^{*} \ni \alpha^{\otimes 3} \longleftrightarrow \sum_{i+j=3} \alpha_{1}^{i} \alpha_{2}^{j} \frac{\partial}{\partial \underbrace{1 \cdots 1}_{i} \underbrace{2 \cdots 2}_{j}}\right|_{m^{2}} \in V_{m^{2}} M^{(2)} .
$$

Even if the sections of $V M$ are not, strictly speaking, vector fields, and a such they lack an immediate geometric interpretation, the bundle $V M$ itself has important relationships with the contact bundle $\mathcal{C}^{1}$. Namely, on one hand, it is canonically embedded into the module of 1 -forms on $\mathcal{C}^{1}$ (see (4.9) later on) and, on the other hand, it is identified with the quotient distribution $\frac{\left(\mathcal{C}^{1}\right)^{\prime}}{\mathcal{C}^{1}}$ (Lemma 4.3 below). 
Lemma 4.3. There is a (conformal) $^{6}$ natural isomorphism

$$
\frac{\left(\mathcal{C}^{1}\right)^{\prime}}{\mathcal{C}^{1}} \cong L^{*}
$$

Proof. It follows from a natural isomorphsim between the left-hand sides of (4.3) and (4.6). Indeed, the map

$$
\frac{\left(\mathcal{C}^{1}\right)_{m^{1}}^{\prime}}{\mathcal{C}_{m^{1}}^{1}} \longrightarrow \frac{\mathcal{C}_{\pi\left(m^{1}\right)}}{L_{m^{1}}}
$$

induced from $\pi_{*}$ is well-defined and linear, for all $m^{1} \in M^{(1)}$. In other words, (4.7) is a bundle morphism, is well-defined and linear, and surjective. Since the ranks of the bundles are the same, it is an isomorphism.

We stress that there is no canonical way to project the bundle $\mathcal{C}^{1}$ over the tautological bundle $L$, if both are understood as bundles over $M^{(2)}$. Nevertheless, if $\mathcal{C}^{1}$ and $L$ are regarded as bundles over $M^{(1)}$, then $\pi_{*}$ turns out to be a bundle epimorphism from $\mathcal{C}^{1}$ to $L$, i.e.,

$$
\pi_{*}: \mathcal{C}^{1} \longrightarrow L=\pi_{*}\left(\mathcal{C}^{1}\right)
$$

Dually, epimorphism (4.8) leads to the bundle embedding $L^{*} \hookrightarrow \mathcal{C}^{1 *}$ which can be combined with the identification $L^{*} \cong V M$. The result is a (conformal) embedding of bundles over $M^{(1)}$,

$$
V M \hookrightarrow \mathcal{C}^{1 *}
$$

which will be useful in the sequel. In local coordinates, (4.9) reads $\left.\partial_{p_{i}}\right|_{m} \longmapsto d_{m^{1}} x^{i}, i=1,2$.

Now we are in position to define the concept of orthogonality in the meta-symplectic context (that has apparently never been observed before), which generalizes the symplectic orthogonality within the contact distribution $\mathcal{C}$ of $M$. Indeed, an immediate consequence of Lemma 4.3 is that the meta-symplectic form $\Omega$ is $L^{*}$-valued, i.e., $\mathcal{C}^{1} \wedge \mathcal{C}^{1} \stackrel{\Omega}{\longrightarrow} L^{*}$ is a trilinear form

$$
\mathcal{C}^{1} \wedge \mathcal{C}^{1} \otimes_{M^{(1)}} L \stackrel{\Omega}{\longrightarrow} C^{\infty}\left(M^{(1)}\right)
$$

In turn, thanks to the canonical projection (4.8) of $\mathcal{C}^{1}$ over $L$, the form (4.10) descends to a trilinear form $\mathcal{C}^{1} \wedge \mathcal{C}^{1} \otimes \mathcal{C}^{1} \stackrel{\widetilde{\Omega}}{\longrightarrow} C^{\infty}\left(M^{(1)}\right)$. Such notions (orthogonal vectors, orthogonal complement, Lagrangian subspaces) can be found also in a 5D meta-symplectic space, but with more subtleties. For example, there can be up to two distinct, so to speak, "orthogonal complements" to a given subspace.

Definition 4.4 (three-fold orthogonality). Elements $X_{1}, X_{2}, X_{3} \in \mathcal{C}^{1}$ are orthogonal if and only if $\widetilde{\Omega}\left(X_{1}, X_{2}, X_{3}\right)=0$.

Remark 4.5. In this perspective, condition (3.6) expresses precisely the fact that the pair $\left\{\mathcal{D}_{2}, \mathcal{D}_{3}\right\}$ is the orthogonal complement of the distribution $\mathcal{D}_{1}$, in the sense that

$$
\widetilde{\Omega}\left(\mathcal{D}_{i}, \mathcal{D}_{j}, \mathcal{D}_{k}\right)=0, \quad\{i, j, k\}=\{1,2,3\} .
$$

\footnotetext{
${ }^{6}$ In the sense that (4.6) is canonical only up to a conformal factor.
} 


\subsection{Rank-one lines, characteristic directions and characteristic hyperplanes}

Isomorphism (4.2) shows that there is a canonical distinguished subset of tangent directions to $M_{m^{k-1}}^{(k)}, k \in\{1,2\}$, namely those sitting in the image of the Veronese embedding $\mathbb{P} L^{*} \hookrightarrow$ $\mathbb{P} S^{k+1} L^{*}$, i.e., the $k^{\text {th }}$ powers of sections of $L^{*}$. Geometrically, these are the tangent directions at $m^{k}=m^{k}(0)$ to the curves $m^{k}(t)$ such that the corresponding family $L_{m^{k}(t)}$ of Lagrangian subspaces in $M^{(k-1)}$ "rotates" around a common hyperplane (which, in our case, is a line).

Local sections of the dual bundle $\mathcal{C}^{k *}$ will be called vertical forms, as they are the image of the local sections of $V^{*} M^{(k)}$ under the natural projection ${ }^{7}$. Because of the dual of the fundamental isomorphism (see Lemma 4.1), $\mathcal{C}^{k *}$ is the epimorphic image of the symmetric power $S^{k+1} L$ of the tautological bundle, and we can speak of decomposable vertical forms, if they come from exact powers.

Definition 4.6. A line $\ell \in \mathbb{P} V_{m^{k}} M^{(k)}$ is called a rank-one line if $\ell=\left\langle\alpha^{\otimes(k+1)}\right\rangle$, for some $\langle\alpha\rangle \in \mathbb{P} L_{m^{k}}^{*}$, in which case we call $\alpha$ a characteristic covector and $\langle\alpha\rangle$ a characteristic (direction) in the point $m^{k}$. The subspace $H_{\alpha}:=\operatorname{ker} \alpha \leq L_{m^{k}}$ is called the characteristic hyperplane associated to the rank-one line $\ell$. Furthermore, if $\omega \in \mathcal{C}^{k *}$ is a vertical form, a hyperplane $H_{\alpha}$ is called characteristic for $\omega$ if $\left.\omega\right|_{\ell}=0$.

Denomination rank-one refers to the rank of the multi-linear symmetric form on $L$ involved in the definition (see, e.g., [13]). Observe that, in our contest, $\operatorname{dim} H_{\alpha}=1$, so that we shall speak of a characteristic line and call characteristic vector a generator of $H_{\alpha}$. The geometric relationship between $H_{\alpha}$ and $\ell=\left\langle\alpha^{\otimes 3}\right\rangle$ is well-known (see, e.g., [29]) and it can be rendered by

$$
\ell=\mathbb{P} T_{m^{k}}\left(H_{\alpha}^{(1)}\right)
$$

where the $1^{\text {st }}$ prolongation $H_{\alpha}^{(1)}=\left\{m^{k} \in M_{m^{k-1}}^{(k)} \mid L_{m^{k}} \supseteq H_{\alpha}\right\}$ of $H_{\alpha}$ has dimension one.

By prolongation of a subspace $W \subseteq T_{m^{k}} M^{(k)}$ we mean the subset $W^{(1)} \subseteq M_{m^{k}}^{(k+1)}$ made of the Lagrangian planes which are contained in $W$. The prolongation of a submanifold $\mathcal{W} \subseteq M^{(k)}$ is the sub-bundle of $\mathcal{W}^{(1)} \subseteq M^{(k+1)}$ defined by $\mathcal{W}_{m^{k}}^{(1)}:=\left(T_{m^{k}} \mathcal{W}\right)^{(1)}, m^{k} \in M^{(k)}$. The prolongation to $M^{(k)}$ of a local contactomorphism $\psi$ of $M$ is denoted by $\psi^{(k)}$.

Remark 4.7. Let $\alpha$ and $L_{m^{2}}$ be locally described by (4.4) and (3.12). Then $H_{\alpha}=\operatorname{ker}\left(\alpha_{1} d x^{1}+\right.$ $\left.\alpha_{2} d x^{2}\right)=\left\langle\alpha_{2} \xi_{1},-\alpha_{1} \xi_{2}\right\rangle$, i.e., one can identify $\alpha \leftrightarrow H_{\alpha}$ via a counterclockwise $\frac{\pi}{2}$ rotation:

$$
\alpha \equiv\left(\alpha_{1}, \alpha_{2}\right) \leftrightarrow\left(\alpha_{2},-\alpha_{1}\right) \equiv H_{\alpha}
$$

From now on, characteristic directions and characteristic lines will be taken as synonyms, thus breaking the separation proposed below:

\begin{tabular}{c|c|c} 
& contravariant object & covariant object \\
\hline$M^{(2)}$ & $\begin{array}{c}\ell=T_{m^{2}} H_{\alpha}^{(1)} \\
\text { rank-one line }\end{array}$ & \\
\hline$M^{(1)}$ & $\begin{array}{c}H_{\alpha}=\operatorname{ker} \alpha \in \mathbb{P} L_{m^{2}} \\
\text { characteristic hyperplane=line }\end{array}$ & $\begin{array}{l}\langle\alpha\rangle \in \mathbb{P} L_{m^{2}}^{*}, \quad \alpha \in L_{m^{2}}^{*} \backslash\{0\} \\
\text { characteristic direction characteristic covector }\end{array}$
\end{tabular}

It should be stressed that in the multidimensional cases (see, e.g., [2]) it is no longer possible to regard the characteristic directions as lines lying in $L$, since the latter are not hyperplanes.

\footnotetext{
${ }^{7}$ We insist that "vertical forms" are not, globally speaking, differential forms on $M^{(k)}$, but rather equivalence classes of them. Locally, of course, one can find a representative in each class.
} 


\subsection{Canonical directions associated with orthogonal distributions}

Suppose that the vertical form $\omega \in \mathcal{C}^{1 *}$ is decomposable in the sense of Lemma 4.1. Then it is easy to see that there are two characteristic lines $H_{1}$ and $H_{2}$. The meta-symplectic form (3.1) links these two lines with the vertical distribution $V:=\operatorname{ker} \omega$ determined by $\omega$.

Recall now that $\Omega$ is $V M \simeq L^{*}$-valued (see (4.2) and (4.10)), so that $H_{i}$ can be considered as a subspace of $V M$ (in view of Remark 4.7), and there are the induced maps

$$
\Omega(X, \cdot): \mathcal{C}^{1} \longrightarrow \frac{V M}{H_{i}}, \quad X \in \mathcal{C}^{1}
$$

Letting $X$ vary in $V$, one gets the rank $\leq 2$ sub-bundle

$$
\frac{V\lrcorner \Omega}{H_{i}}:=\left\langle\Omega(X, \cdot)+H_{i} \mid X \in V\right\rangle \subset \mathcal{C}^{1 *} \otimes \frac{V M}{H_{i}} .
$$

Finally, $H_{j} \otimes \frac{L^{*}}{H_{i}}$ can be regarded as a one-dimensional sub-bundle of $\mathcal{C}^{1 *} \otimes \frac{V M}{H_{i}}$, via (4.9).

Taking this into account, we have the following result.

Lemma 4.8. It holds the following identification

$$
\frac{V\lrcorner \Omega}{H_{i}} \simeq H_{j} \otimes \frac{L^{*}}{H_{i}}, \quad\{i, j\}=\{1,2\}
$$

In particular, $\operatorname{dim} \frac{V\lrcorner \Omega}{H_{i}}=1, i=1,2$.

Proof. We shall assume that the coefficient of $d p_{11}$ is not zero: other cases can be dealt with likewise. So, being $\omega$ decomposable, it can be brought, up to a scaling, to the form $\omega=d p_{11}-\left(k_{1}+k_{2}\right) d p_{12}+k_{1} k_{2} d p_{22} \in \mathcal{C}^{1 *}$. Accordingly, $H_{i}=\partial_{p_{2}}+k_{i} \partial_{p_{1}}$ and a straightforward computation shows that $V=\left\langle X_{1}, X_{2}\right\rangle$, with

$$
X_{1}=-k_{1} k_{2} \partial_{p_{11}}+\partial_{p_{22}}, \quad X_{2}=\left(k_{1}+k_{2}\right) \partial_{p_{11}}+\partial_{p_{12}} .
$$

Then

$$
\begin{aligned}
V\lrcorner \Omega & \left.\left.=\left\langle X_{1}\right\lrcorner \Omega, X_{2}\right\lrcorner \Omega\right\rangle \\
& =\left\langle k_{1} k_{2} d x^{1} \otimes \partial_{p_{1}}-d x^{2} \otimes \partial_{p_{2}},-\left(k_{1}+k_{2}\right) d x^{1} \otimes \partial_{p_{1}}-d x^{1} \otimes \partial_{p_{2}}-d x^{2} \otimes \partial_{p_{1}}\right\rangle .
\end{aligned}
$$

If the basis $\left\{H_{i}, \partial_{p_{1}}\right\}$ for $V M \simeq L^{*}$ (see (4.2)) is chosen, then the factor of (4.14) with respect to $H_{i}$ is the line

$$
\left\langle\left(k_{j} d x^{1}+d x^{2}\right) \otimes \partial_{p_{1}}\right\rangle, \quad i \neq j,
$$

and the lemma is proved.

Lemma 4.8 will be used later on in the proof of Proposition 6.7, establishing that, associated with a quasi-linear MAE with completely decomposable symbol, there are three canonical lines in $M$.

\subsection{Some examples}

Let $\mathcal{E}$ be as in (1.1), with $F$ given by $p_{111}-p_{112}-2 p_{122}$ (resp., $p_{122}$ and $p_{111}$ ). Then equation (1.7) reads

$$
\left(\nu^{2}\right)^{3}+\left(\nu^{2}\right)^{2} \nu^{1}-2 \nu^{2}\left(\nu^{1}\right)^{2}=0 \quad\left(\text { resp., } \nu^{2}\left(\nu^{1}\right)^{2}=0 \text { and }\left(\nu^{2}\right)^{3}=0\right) .
$$


Applying definition (1.9), one obtains that $\mathcal{V}^{\mathcal{E}}=\cup_{i=1}^{3} \mathbb{P} \mathcal{D}_{i}$, with

$$
\begin{aligned}
& \mathcal{D}_{1}=\left\langle D_{1}, \partial_{p_{11}}+\partial_{p_{12}}, 2 \partial_{p_{11}}+\partial_{p_{22}}\right\rangle, \\
& \mathcal{D}_{2}=\left\langle D_{1}+D_{2}, 2 \partial_{p_{11}}+\partial_{p_{12}}, \partial_{p_{22}}\right\rangle \\
& \mathcal{D}_{3}=\left\langle D_{1}-2 D_{2}, \partial_{p_{11}}-\partial_{p_{12}}, \partial_{p_{22}}\right\rangle
\end{aligned}
$$

(resp.,

$$
\mathcal{D}_{1}=\left\langle D_{1}, \partial_{p_{11}}, \partial_{p_{12}}\right\rangle, \quad \mathcal{D}_{2}=\mathcal{D}_{3}=\left\langle D_{2}, \partial_{p_{11}}, \partial_{p_{22}}\right\rangle
$$

and $\left.\mathcal{D}_{1}=\mathcal{D}_{2}=\mathcal{D}_{3}=\left\langle D_{1}, \partial_{p_{12}}, \partial_{p_{22}}\right\rangle\right)$. Observe that, in the first case, equation (4.16) factors as

$$
\nu^{2}\left(\nu^{2}-\nu^{1}\right)\left(\nu^{2}+2 \nu^{1}\right)=0,
$$

and that the "horizontal components" $h_{i}$ (i.e., the first generators) of (4.17), (4.18) and (4.19) are precisely the three distinct roots of (4.21). Notice also that, in general, the distributions associated with the same quasi-linear $3^{\text {rd }}$ order MAE need not to be contactomorphic. Here it follows an easy counterexample. For instance, consider the equation $p_{122}=0$ above: its characteristic cone consists of the two distributions $\mathcal{D}_{1}$ and $\mathcal{D}_{2}=\mathcal{D}_{3}$ (see (4.20)), which are not contactomorphic. Indeed, the derived distribution $\mathcal{D}_{1}^{\prime}:=\mathcal{D}_{1}+\left[\mathcal{D}_{1}, \mathcal{D}_{1}\right]$ of $\mathcal{D}_{1}$ is 5-dimensional, whereas $\operatorname{dim}\left(\mathcal{D}_{2}^{\prime}\right)=4$.

Let $\mathcal{E}$ be the first equation from above, i.e., with $F=p_{111}-p_{112}-2 p_{122}$. The following three lines

$$
\begin{aligned}
1^{\text {st }} \text { line: } & \Omega\left(\mathcal{D}_{1}, \mathcal{D}_{2}\right)=\left\langle 2 \partial_{p_{1}}+\partial_{p_{2}}\right\rangle, \\
2^{\text {nd }} \text { line: } & \Omega\left(\mathcal{D}_{1}, \mathcal{D}_{3}\right)=\left\langle\partial_{p_{1}}-\partial_{p_{2}}\right\rangle, \\
3^{\text {rd }} \text { line: } & \Omega\left(\mathcal{D}_{2}, \mathcal{D}_{3}\right)=\left\langle\partial_{p_{2}}\right\rangle,
\end{aligned}
$$

are canonically associated with the triple $\left(\mathcal{D}_{1}, \mathcal{D}_{2}, \mathcal{D}_{3}\right)$, i.e., with the equation $\mathcal{E}$. It is worth observing that the vertical part $\mathcal{D}_{1}^{v}$ is an integrable vertical distribution on $M^{(1)}$, so that, in this case, lines (4.23) and (4.24) are the characteristic lines of the family of equations $2 p_{22}-p_{11}+p_{12}=$ $k\left(x^{1}, x^{2}, u\right)$. Nevertheless, integrability $\mathcal{D}_{1}^{v}$ is not indispensable for associating directions (4.23) and (4.24) with the distribution $\mathcal{D}_{1}$, since they occur as the characteristic lines of the (not necessarily closed) vertical covector $2 d p_{22}-d p_{11}+d p_{12}$, which annihilates $\mathcal{D}_{1}^{v}$.

Now we give another example that clarifies the type-changing phenomenon:

$$
p_{11}\left(p_{112} p_{122}-p_{111} p_{222}\right)-p_{122}=0 .
$$

In fact, equation (4.25) is non-linear at the points with $p_{11} \neq 0$, whereas it is linear at the points with $p_{11}=0$. Equation (4.25) is of Goursat type and the corresponding 3-dimensional distribution

$$
\mathcal{D}=\left\langle D_{1}, p_{11} D_{2}+\partial_{p_{11}}, \partial_{p_{12}}\right\rangle
$$

behaves accordingly with Theorem 3.5, i.e., the dimension of $\mathcal{D}^{v}$ is 1 if $p_{11} \neq 0$ and it is 2 if $p_{11}=0$. Moreover, at the points with $p_{11}=0$ we have three planes, whose two of them coincide, defining the fibre of equation (4.25) (see (4.20)).

\subsection{Smoothness and singularities issues}

For generic PDEs, we just assume that $\mathcal{E} \longrightarrow M^{(1)}$ is the zero locus of a smooth function, without requiring the non-vanishing of its differential. In all the statements and reasonings, we tacitly restrict ourselves to the open and dense subset $\mathcal{E}_{\text {reg }}$ of smooth points. 
According to our definition of a $\operatorname{PDE} \mathcal{E}$ as a sub-bundle of $M^{(1)}$, the natural projection $\mathcal{E} \longrightarrow M^{(1)}$ is surjective, and that each fibre $\mathcal{E}_{m^{1}}$ is a smooth submanifold of $M_{m^{1}}^{(2)}$, possibly with singularities.

For example, the singularities which can occur for $3^{\text {rd }}$ Monge-Ampère equations are of "algebraic type", i.e., $\mathcal{E} \longrightarrow M^{(1)}$ is a bundle of projective varieties. Indeed, each fibre $M_{m^{1}}^{(2)}$ is naturally understood as the Grassmannian variety of isotropic elements with respect to the metasymplectic structure which, in analogy with the standard Lagrangian Grassmannian, embeds into a suitable projective space via the Plücker embedding ${ }^{8}$. More precisely, a straightforward computation shows that the subset of the singular points of $\mathcal{E}_{\mathcal{D}}$ is made by the $\mathrm{m}^{2}$ 's such that $L_{m^{2}} \subset \mathcal{D}_{m^{1}}$. Such a possibility occurs only when $\operatorname{dim}\left(L_{m^{2}} \cap \mathcal{D}_{m^{1}}\right)=2$ for $m^{2} \in M^{(2)}$ (in the case when $L_{m^{2}}$ and $\mathcal{D}_{m^{1}}$ are not transversal, generically the dimension is 1 , otherwise is generically 0$)$.

Methodologically, this paper is at the crossroad between algebraic and differential geometry. The geometric approach to non-linear PDEs is traditionally differential, while recent developments revealed that many features of PDEs are genuinely algebraic-geometric. Even though a rigorous algebraic-geometric approach to the present topics is feasible, the authors have opted for the traditional and - in a sense, easier - methods, based on elementary differential geometry.

Summing up, all the objects are herewith assumed to be smooth, and all maps to be of class $C^{\infty}$, except for:

- the fibres of $\mathcal{E}$, which are the zero loci of smooth functions (and, as such, may display singularities where the differential vanishes);

- the sub-bundles $\mathcal{V}$ of the projectivised contact bundle $\mathbb{P C}$, which are smooth families of projective sub-varieties in each fibre, i.e., locally isomorphic to the product of an open subset of $\mathcal{V}$ by a projective subvariety in $\mathbb{P}^{4}$.

If $\mathcal{V}_{\mathrm{I}}$ and $\mathcal{V}_{\mathrm{II}}$ are sub-bundles of $\mathcal{V}$, in the aforementioned sense, we say that $\mathcal{V}_{\mathrm{I}}$ and $\mathcal{V}_{\mathrm{II}}$ are irreducible components of $\mathcal{V}$ if, for any point $m^{1} \in M^{(1)}$, the projective variety $\mathcal{V}_{m^{1}}$ is reducible, and $\left(\mathcal{V}_{\mathrm{I}}\right)_{m^{1}}$ and $\left(\mathcal{V}_{\mathrm{II}}\right)_{m^{1}}$ are its irreducible components.

We conclude this preliminary part of the paper by observing that, locally, $M^{(k)}$ is the $k+1^{\text {st }}$ jet-extension of the trivial bundle $\mathbb{R}^{2} \times \mathbb{R} \rightarrow \mathbb{R}^{2}$, so that the reader more at ease with jet formalism may perform the substitution

$$
M^{(k)} \longleftrightarrow J^{k+1}(2,1), \quad k \geq 0,
$$

and easily adapt the main results to the new setting.

\section{Characteristics of $3^{\text {rd }}$ order PDEs}

Now we turn our attention to the hypersurfaces in $M^{(2)}$ that play the role of $3^{\text {rd }}$ order PDEs in our analysis.

Definition 5.1. A characteristic covector $\alpha \in L_{m^{2}}^{*} \backslash\{0\}$, with $m^{2} \in \mathcal{E}_{m^{1}}$, is characteristic for $\mathcal{E}$ in $m^{2}$ if the corresponding rank-one line $\ell$ is tangent to $\mathcal{E}_{m^{1}}$ in $m^{2}$, in which case $\langle\alpha\rangle$ is a characteristic (direction) for $\mathcal{E}$ in $m^{2}$ and the subspace $H_{\alpha}$ is a characteristic hyperplane for $\mathcal{E}$.

Formula (4.11) says precisely that the rank-one line $\ell$ which corresponds to $\left\langle\alpha^{\otimes 3}\right\rangle$ via the isomorphism (4.2) is precisely the (one-dimensional) tangent space to the prolongation $H_{\alpha}^{(1)}$. In this perspective, $\langle\alpha\rangle$ is a characteristic for $\mathcal{E}$ at $m^{2}$ if and only if $H_{\alpha}^{(1)}$ is tangent to $\mathcal{E}_{m^{1}}$ at $m^{2}$ but, in general, $H_{\alpha}^{(1)}$ does not need to touch $\mathcal{E}_{m^{1}}$ in any other point.

\footnotetext{
${ }^{8}$ The general theory of this would lead us beyond the scope of the present paper.
} 
Definition 5.2. If $H_{\alpha}^{(1)}$ is entirely contained in $\mathcal{E}_{m^{1}}$, then $\langle\alpha\rangle$ is called a strong characteristic (direction) and $H_{\alpha}$ a strongly characteristic line (in $m^{2}$ ).

Example 5.3. Function (1.1) determines, for $k=2$, the $3^{\text {rd }}$ order $\operatorname{PDE} \mathcal{E}=\{F=0\}$. Now equation (1.7) can be correctly interpreted as follows: it is satisfied if and only if the vector $\nu=\nu^{i} \xi_{i}$ given by (1.8) spans a characteristic line of $\mathcal{E}$. The very same equation (1.7) tells also when the covector $\alpha=\nu^{2} d x^{1}-\nu^{1} d x^{2}$ spans a characteristic direction of $\mathcal{E}$. In the last perspective, equation (1.7) is nothing but the right-hand side of (4.5) applied to $F$ and equated to zero.

\subsection{Characteristics of a $3^{\text {rd }}$ order PDE and relationship with its symbol}

For any $m^{2} \in \mathcal{E}_{m^{1}}$ we define the vertical tangent space to $\mathcal{E}$ at $m^{2}$ as the subspace

$$
V_{m^{2}} \mathcal{E}:=T_{m^{2}} \mathcal{E}_{m^{1}} \leq V_{m^{2}} M^{(2)},
$$

called the symbol of $\mathcal{E}$ by many authors ${ }^{9}$. Obviously, vertical tangent spaces can be naturally assembled into a linear bundle

$$
V \mathcal{E}:=\coprod_{m^{2} \in \mathcal{E}} V_{m^{2}} \mathcal{E}
$$

called the vertical bundle of $\mathcal{E}$. Directly from (5.1) it follows the bundle embedding $V \mathcal{E} \subseteq$ $\left.V M^{(2)}\right|_{\mathcal{E}}$, where fibres of the former are hyperplanes in the fibres of the latter. Thanks to Lemma 4.1, $V_{m^{2}} \mathcal{E}$ can also be regarded as a subspace of $S^{3} L_{m^{2}}^{*}$, and, being the identification (4.2) manifestly conformal, such an inclusion descends to the corresponding projective spaces, i.e.,

$$
\mathbb{P} V_{m^{2}} \mathcal{E} \subseteq \mathbb{P} S^{3} L_{m^{2}}^{*} .
$$

The dual of canonical inclusion (5.2) reads

$$
\operatorname{Ann}\left(V_{m^{2}} \mathcal{E}\right) \in \mathbb{P} S^{3} L_{m^{2}},
$$

where $\operatorname{Ann}\left(V_{m^{2}} \mathcal{E}\right)$ is the (one-dimensional) subspace $\left(V_{m^{2}} M^{(2)}\right)^{*}$ made of covectors vanishing on the hyperplane $V_{m^{2}} \mathcal{E}$, viz.

$$
\operatorname{Ann}\left(V_{m^{2}} \mathcal{E}\right) \leq\left(V_{m^{2}} M^{(2)}\right)^{*}=\left(S^{3} L_{m^{2}}^{*}\right)^{*}=S^{3} L_{m^{2}} .
$$

From a global perspective, (5.3) is nothing but the definition of a section

$$
\mathbb{P} S^{3} L \stackrel{\operatorname{Ann}(V \mathcal{E})}{\longleftrightarrow} \mathcal{E} .
$$

Remark 5.4 (symbol of a function). Let $\mathcal{E}=\{F=0\}$, and use the same coordinates (3.13) of Remak 3.6. Then

$$
\operatorname{Ann}\left(V_{m^{2}} \mathcal{E}\right)=\left\langle\operatorname{Smbl}_{m^{2}} F\right\rangle,
$$

where $\mathrm{Smbl}_{m^{2}} F$ is the symbol of $F$ at $m^{2}$, i.e.,

$$
\mathrm{Smbl}_{m^{2}} F=\left.\frac{\partial F}{\partial p_{i j k}}\right|_{m^{2}} \xi_{i} \xi_{j} \xi_{k}
$$

where $\xi_{i}$ has been defined in (3.12). Observe that $\operatorname{Ann}\left(V_{m^{2}} \mathcal{E}\right)$ is independent of the choice of $F$ in the ideal determined by $\mathcal{E}$, so that $\operatorname{Ann}(V \mathcal{E})$ can be replaced with $\operatorname{Smbl} F$.

\footnotetext{
${ }^{9}$ Herewith we prefer to use the term "symbol" only for the function $F$ determining $\mathcal{E}$, and not for $\mathcal{E}$ itself. The two things, however, are the same, if one just regards $F$ as a the ( $3^{\text {rd }}$ order) differential operator defining the equation $\mathcal{E}$.
} 
In view of (5.4), a direction $\langle\alpha\rangle \in \mathbb{P} L_{m^{2}}^{*}$ is a characteristic one for $\mathcal{E}$ at $m^{2}$ if and only if

$$
\left\langle\mathrm{Smbl}_{m^{2}} F, \alpha^{\otimes 3}\right\rangle=0
$$

where $\langle\cdot, \cdot\rangle$ is the canonical pairing on $S^{3} L_{m^{2}}$. Needless to say, (5.7) is independent of the choice of $\alpha$ (resp., $F$ ) representing $\langle\alpha\rangle$ (resp., $\mathcal{E}$ ). Similarly, $H=\langle\nu\rangle$, with $\nu=\nu^{i} \xi_{i}$, is a characteristic line if and only if

$$
\left.\sum_{i+j=3}(-1)^{i} \frac{\partial F}{\partial \underbrace{p_{i}^{1 \cdots 12 \cdots 2}}_{i}}\right|_{m^{2}}\left(\nu^{1}\right)^{i}\left(\nu^{2}\right)^{j}=0,
$$

which eventually clarifies formula (1.7).

Remark 5.5 (coordinates of $V \mathcal{E}$ ). If $\mathcal{E}=\{F=0\}$ is a $3^{\text {rd }}$ order PDE according to the definition given at the end of Section 2.1, then $\left.d F\right|_{\mathcal{E}_{m^{1}}}$ is nowhere zero, for all $m^{1} \in M^{(1)}$ (recall Section 4.5). So,

$$
V_{m^{2}} \mathcal{E}=\left.\operatorname{ker} \frac{\partial F}{\partial p_{i j k}}\right|_{m^{2}} d p_{i j k}
$$

is an hyperplane in the $4 \mathrm{D}$ space $V_{m^{2}} M^{(2)}$.

Remark 5.6 (types of $3^{\text {rd }}$ order PDEs). Observe that (5.6) is a $3^{\text {rd }}$ order homogeneous polynomial in two variables, so that we can introduce its discriminiant $\Delta=18 a b c d-4 b^{3} d+b^{2} c^{2}-$ $4 a c^{3}-27 a^{2} d^{2}$, where $a, b, c, d \in C^{\infty}\left(M^{(2)}\right)$ are defined by

$$
a\left(m_{2}\right)=\left.\frac{\partial F}{\partial p_{111}}\right|_{m^{2}}, \quad b\left(m_{2}\right)=\left.\frac{\partial F}{\partial p_{112}}\right|_{m^{2}}, \quad c\left(m_{2}\right)=\left.\frac{\partial F}{\partial p_{122}}\right|_{m^{2}}, \quad d\left(m_{2}\right)=\left.\frac{\partial F}{\partial p_{222}}\right|_{m^{2}} .
$$

Thus, any point $m_{2} \in \mathcal{E}$ can be of three different types, according to the signature of $\Delta$, namely,

- $\Delta\left(m_{2}\right)>0 \Leftrightarrow(5.6)$ decomposes into three distinct linear factors;

- $\Delta\left(m_{2}\right)=0 \Leftrightarrow(5.6)$ contains the square of a linear factor;

- $\Delta\left(m_{2}\right)<0 \Leftrightarrow(5.6)$ contains an irreducible quadratic factor.

Equation $\mathcal{E}=\{F=0\}$ may be called "fully parabolic" in $m^{2} \in \mathcal{E}$ if (5.6) reduces to the cube of linear factor in the point $m^{2}$.

\subsection{The characteristic variety as a covering of the characteristic cone}

Let $\mathcal{E}=\{F=0\}$, and regard $L$ as a sub-bundle of the pull-back of $\mathcal{C}^{1}$ to the equation $\mathcal{E}$, bearing in mind the diagram
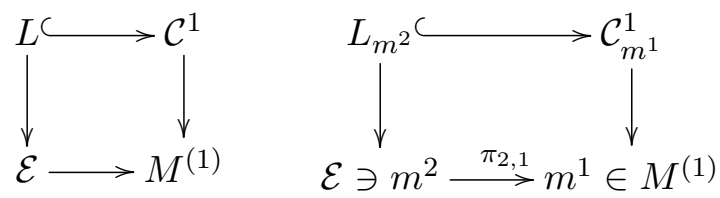

Now we can come back to the direction $\nu$ defined by (1.8) and notice that it lies in $L_{\bar{m}^{2}}$, and also provide an intrinsic way to check whether $\nu$ is characteristic or not. Indeed, in view of the fundamental isomorphism (4.2), one can regard the cube $\nu^{\otimes 3}$ of $\nu$ as a tangent vector to the fibre $M_{\bar{m}^{2}}^{(2)}$, and equation (1.7) tells precisely when $\nu^{\otimes 3}$ belongs to the sub-space $V_{\bar{m}^{2}} \mathcal{E}=T_{\bar{m}^{2}} \mathcal{E}_{\bar{m}^{1}}$ 
(see (5.1)), up to a line-hyperplane duality (see also Remark 4.7). In other words, the set of characteristic lines

$$
\operatorname{char}_{m^{2}}^{\mathbb{R}} \mathcal{E}:=\left\{H \in \mathbb{P} L_{m^{2}}^{*} \mid H \text { is a characteristic hyperplane of } \mathcal{E}\right\} \subseteq \mathbb{P} L_{m^{2}}^{*}
$$

is the projective sub-variety in $\mathbb{P} L_{m^{2}}^{*}$ cut out by (1.7).

Since we plan to carry out an analysis of certain PDEs via their characteristics, it seems natural to consider all characteristic lines at once, i.e., as a unique geometric object. Traditionally, one way to accomplish this is to take the disjoint union

$$
\operatorname{char}^{\mathbb{R}} \mathcal{E}:=\coprod_{m^{2} \in \mathcal{E}} \operatorname{char}_{m^{2}}^{\mathbb{R}} \mathcal{E}
$$

known as the characteristic variety of $\mathcal{E}$ (see, e.g., [11]). As a bundle over $\mathcal{E}$, the family of the fibres of $\operatorname{char}^{\mathbb{R}} \mathcal{E}$ coincides with the equation $\mathcal{E}$ itself. So, the bundle char ${ }^{\mathbb{R}} \mathcal{E}$, in spite of its importance for the study of a given equation $\mathcal{E}$, cannot be used to define $\mathcal{E}$ as an object pertaining to $M^{(1)}$. Still this can be arranged: it suffices "to project everything one step down", so to speak. The result is precisely the characteristic cone $\mathcal{V}^{\mathcal{E}}$ :

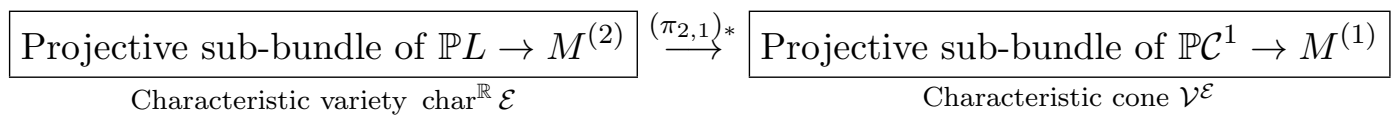

It is easy to see that some characteristic lines, which are distinct entities in $\operatorname{char}^{\mathbb{R}} \mathcal{E}$, may collapse into $\mathcal{V}^{\mathcal{E}}$ (e.g., when the corresponding fibres of the tautological bundle have non-zero intersection): hence, $\mathcal{V}^{\mathcal{E}}$ is not the most appropriate environment for the study of characteristics. On the other hand, $\mathcal{V}^{\mathcal{E}}$ is a bundle over $M^{(1)}$, where there is no trace of the original equation $\mathcal{E}$ : as such, it may effectively replace the equation itself and serves as a source of its invariants.

Now we can give a rigorous definition of our main tool, the characteristic cone of $\mathcal{E}$. Indeed (see Remark 4.7), the characteristic variety $\operatorname{char}^{\mathbb{R}} \mathcal{E}$ can be regarded as a sub-bundle of $\mathbb{P} L \longrightarrow \mathcal{E}$. In turn, this makes it possible to use the commutative diagram (5.8) to map $\operatorname{char}^{\mathbb{R}} \mathcal{E}$ to $\mathbb{P C}^{1}$ and define the sub-bundle

$$
\begin{aligned}
\mathcal{V}^{\mathcal{E}}:=\{ & H \in \mathbb{P C}^{1} \mid \exists m^{2} \in \mathcal{E}: H \subset L_{m^{2}} \text { and } \\
& \left.H \text { is a characteristic hyperplane of } \mathcal{E} \text { at } m^{2}\right\} \subseteq \mathbb{P C}^{1}
\end{aligned}
$$

as the image of such a mapping.

Definition 5.7. Above defined bundle $\mathcal{V}^{\mathcal{E}} \longrightarrow M^{(1)}$ is the characteristic cone of $\mathcal{E}$.

By its definition, $\mathcal{V}^{\mathcal{E}}$ fits into the commutative diagram

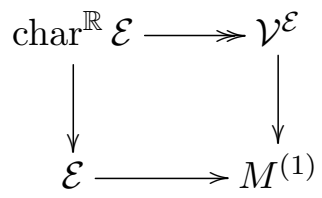

revealing that, in a sense, $\mathcal{V}^{\mathcal{E}}$ is covered by $\operatorname{char}^{\mathbb{R}} \mathcal{E}$.

It is worth observing that, from a local perspective, (1.9), (5.9), and Definition 5.7 all define the same object. For instance, by Definition 5.7, a line $H=\langle\nu\rangle \in \mathbb{P C}_{m^{1}}^{1}$ belongs to $\mathcal{V}_{m^{1}}^{\mathcal{E}}$ if and only if there is a point $m^{2} \in \mathcal{E}_{m^{1}}$ such that $H$ is a characteristic line for $\mathcal{E}$ at the point $m^{2}$. But Example 5.3 shows that this is the case if and only if the generator $\nu=\left(\nu^{1}, \nu^{2}\right)$ of $H$ satisfies equation (1.7). 


\subsection{The irreducible component $\mathcal{V}_{\mathrm{I}}^{\mathcal{E}}$ of the characteristic cone of a $3^{\text {rd }}$ order PDE $\mathcal{E}$}

Let $\mathcal{E}=\{F=0\}$ and identify $\operatorname{Ann}(V \mathcal{E})$ with $\operatorname{Smbl} F$ as in Remark 5.4. As a homogeneous cubic tensor on $L$ (see (5.5)), Smbl $F$ possesses a linear factor, i.e., a section

$$
\mathbb{P} L \stackrel{\mathrm{Smbl}_{I} F}{\longleftrightarrow} \mathcal{E}
$$

such that

$$
\operatorname{Smbl} F=\operatorname{Smbl}_{I} F \odot \operatorname{Smbl}_{I I} F
$$

where $\operatorname{Smbl}_{I I} F$ is a section of $\mathbb{P} S^{2} L \rightarrow \mathcal{E}$.

Plugging (5.11) into (5.7) shows that, in order to have

$$
\left\langle\operatorname{Smbl}_{I, m^{2}} F \odot \operatorname{Smbl}_{I I, m^{2}} F, \alpha \odot \alpha^{2}\right\rangle=\left\langle\operatorname{Smbl}_{I, m^{2}} F, \alpha\right\rangle\left\langle\operatorname{Smbl}_{I I, m^{2}} F, \alpha^{2}\right\rangle=0
$$

it suffices that

$$
\left\langle\operatorname{Smbl}_{I, m^{2}} F, \alpha\right\rangle=0 .
$$

In other words, if (5.12) is satisfied, then $\langle\alpha\rangle \in \mathbb{P} L_{m^{2}}^{*}$ is a characteristic direction (incidentally revealing that $3^{\text {rd }}$ order PDEs always possess a lot of them).

Moreover, in view of the identification of characteristic directions with characteristic lines (Remark 4.7), (5.12) shows also that the line $\operatorname{Smbl}_{I, m^{2}} F \in \mathbb{P} L_{m^{2}}$ is always a characteristic line for $\mathcal{E}$, since there always is an $\alpha$ such that (5.12) is satisfied.

It is clear now how diagram (5.8) can be made use of and how to define, much as we did in Section 4.2 , a subset $\mathcal{V}_{\mathrm{I}}^{\mathcal{E}} \subseteq \mathcal{V}^{\mathcal{E}}$ fitting into the commutative diagram

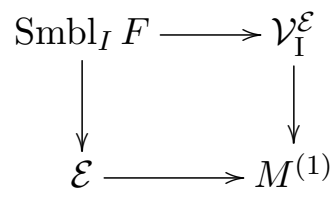

parallel to (5.10). This gives a solid background to the statement of Theorem 3.4, where $\mathcal{V}_{\mathrm{I}}^{\mathcal{E}}$ was mentioned without exhaustive explanations.

\section{Proof of the main results}

Now we are in position to prove the main results, Theorem 3.3, concerned with the structure of $3^{\text {rd }}$ order MAEs, Theorem 3.4 and Theorem 3.5, concerned with the structure of Goursat-type $3^{\text {rd }}$ order MAEs. Since the former are special instances of the latter, we prefer to clarify their relationship beforehand.

\subsection{Reconstruction of PDEs by means of their characteristics}

In spite of its early introduction (1.9), the main object of our interest has been defined again in a less direct way, which passes through the characteristic variety (see above Definition 5.7). The reason behind this choice is revealed by diagram (5.10): the characteristic variety char $^{\mathbb{R}} \mathcal{E}$ plays the role of a "minimal covering object" for both the equation $\mathcal{E}$ itself and its characteristic cone $\mathcal{V}^{\mathcal{E}}$. 
It is easy to guess ${ }^{10}$ that such a minimal covering must be defined in terms of $\Omega$-isotropic flags on $M^{(1)}$, in the sense of Section 2.2 , i.e., by means of the commutative diagram

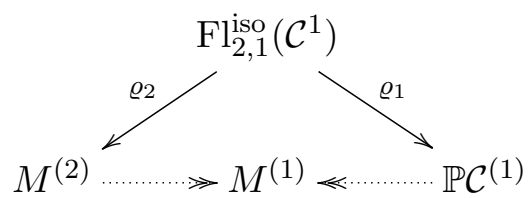

where $\mathrm{Fl}_{2,1}^{\text {iso }}\left(\mathcal{C}^{1}\right):=\left\{\left(m^{2}, H\right) \in M^{(2)} \times{ }_{M^{(1)}} \mathbb{P C}^{1} \mid L_{m^{2}} \supset H\right\}$ is the flag bundle of $\Omega$-isotropic elements of $\mathcal{C}^{1}$.

The "double fibration transform" associated to the diagram (6.1) allows to pass from a subbundle of $M^{(2)}$ (e.g., a $3^{\text {rd }}$ order $\mathrm{PDE} \mathcal{E}$ ) to a sub-bundle of $\mathbb{P C}^{1}$ (e.g., its characteristic cone $\mathcal{V}^{\mathcal{E}}$ ), and vice-versa. Lemma 6.1 indicates how to proceed in one direction.

Lemma 6.1. Let $\mathcal{E}$ be a $P D E$.

1. Take the pre-image $\varrho_{2}^{-1}(\mathcal{E})$ of $\mathcal{E}$ and select the points of tangency with the $\varrho_{1}$-fibres: the result is $\operatorname{char}^{\mathbb{R}} \mathcal{E}$.

2. Project char $^{\mathbb{R}} \mathcal{E}$ onto $\mathbb{P C}^{1}$ via $\varrho_{1}$ : the result is $\mathcal{V}^{\mathcal{E}}$.

Proof. Item 1 follows from the fact that the $\varrho_{1}$-fibres are the prolongations $H^{(1)}$ of hyperplanes $H \in \mathbb{P C}^{(1)}$, and the tangency condition means that they are determined by characteristics (see (4.11)). Item 2 is just a paraphrase of Definition 5.7.

Coming back is easier. Indeed, given a sub-bundle $\mathcal{V} \subseteq \mathbb{P C}^{1}$, the same definition (3.2) of $\mathcal{E}_{\mathcal{V}}$ given earlier can be recast as

$$
\mathcal{E}_{\mathcal{V}}=\varrho_{2}\left(\varrho_{1}^{-1}(\mathcal{V})\right)
$$

In spite of the name "double fibration transform", performing (6.2) first, and then applying Lemma 6.1 to the resulting equation $\mathcal{E}_{\mathcal{V}}$, does not return, as a rule, the original sub-bundle $\mathcal{V}$.

Corollary 6.2. Let $\mathcal{V} \subseteq \mathbb{P C}^{1}$ be a sub-bundle. Then

$$
\mathcal{V} \subseteq \mathcal{V}^{\mathcal{E}_{\mathcal{V}}}
$$

Proof. Directly from Lemma 6.1 and formula (6.2).

We stress that, for any odd-order $\mathrm{PDE} \mathcal{E}$, it holds the inclusion

$$
\mathcal{E} \subseteq \mathcal{E}_{\mathcal{V}^{\mathcal{E}}}
$$

This paper begins to tackle the problem of determining those PDEs which are recoverable from their characteristics in the sense that (3.3) is valid. Lemma 6.3 below provides a simple evidence that, as a matter of fact, not all PDEs are recoverable (in the sense of Definition 3.2).

Lemma 6.3. $\mathcal{V}$ is made of strongly characteristic lines for $\mathcal{E}_{\mathcal{V}}$

Proof. Let $H \in \mathcal{V}$ be a line belonging to $\mathcal{V}$. Then $\varrho_{2}\left(\varrho_{1}^{-1}(H)\right)$ is nothing but $H^{(1)}$ (see Definition 5.2) and (6.2) tells precisely that $H^{(1)}$ is entirely contained in $\mathcal{E}_{\mathcal{V}}$. Hence, $H$ is a strongly characteristic line for $\mathcal{E}_{\mathcal{V}}$ in any point of $\varrho_{2}\left(\varrho_{1}^{-1}(H)\right)$.

\footnotetext{
${ }^{10}$ The structural relationship between the rank-one cone and the characteristic variety, which is the backbone of the present paper, made its appearance in many discussions concerning the characteristic variety, including [11, Chapter V], the late-1960's work by Guillemin on the characteristics of Pfaffian systems, and the more more recent work by Malgrange on the homological algebra of characteristic varieties.
} 
The meaning of Theorem 3.4 is that $3^{\text {rd }}$ order MAEs of Goursat-type are, in a sense, those PDEs whose characteristic cone takes the simplest form, namely that of a linear projective subbundle, and, moreover, they are also recoverable from it. Observe that (3.4) is a particular case of (6.2), so that Goursat-type $3^{\text {rd }}$ order MAEs constitute a remarkable example of equations determined by a projective sub-bundle of $\mathbb{P C}^{1}$. Then Lemma 6.3 reveals that all the lines lying in $\mathcal{D}$ are strongly characteristic lines for $\mathcal{E}_{\mathcal{D}}$, in strict analogy with the classical case [2].

\subsection{Proof of Theorem 3.3}

As we already underlined, we called " 3 rd order MAEs" the equations (3.1) defined by means of a 2 -form, disregarding the fact that, actually, Boillat introduced them by imposing the condition of complete exceptionality (which also allowed him to write down the local form (3.15)).

Fix now a point $m^{1} \in M^{(1)}$ and recall (see Lemma 4.1) that the fibre $M_{m^{1}}^{(2)}$ is an affine space modeled over $S^{3} L_{m^{1}}^{*}$. Let $\omega \in \Lambda^{2} \mathcal{C}^{1 *}$ and regard the corresponding 2 -form $\omega_{m^{1}}$ on $\mathcal{C}_{m^{1}}^{1}$ as a linear map $\Lambda^{2} \mathcal{C}_{m^{1}}^{1} \stackrel{\phi}{\longrightarrow} \mathbb{R}$. The linear projective subspace

$$
\mathcal{H}:=\mathbb{P} \operatorname{ker} \phi \subset \mathbb{P} \Lambda^{2} \mathcal{C}_{m^{1}}^{1}
$$

is the so-called hyperplane section determined by $\omega_{m^{1}}$. Now we show that $\mathcal{H}$ corresponds precisely to the fibre $\mathcal{E}_{\omega, m^{1}}$ of the $3^{\text {rd }}$ order MAE determined by $\omega$, via the Plücker embedding.

Indeed, $S^{3} L_{m^{1}}^{*}$ is embedded into the space $L_{m^{1}}^{*} \otimes S^{2} L_{m^{1}}^{*}$ of all, i.e., not necessarily Lagrangian, 2D horizontal subspaces, via the polarisation/Spencer operator. In turn, $L_{m^{1}}^{*} \otimes S^{2} L_{m^{1}}^{*}$ is an affine neighborhood of $L_{m^{1}}$ in the Grassmannian $\operatorname{Gr}\left(2, \mathcal{C}_{m^{1}}^{1}\right)$, which is sent to $\mathbb{P} \Lambda^{2} \mathcal{C}_{m^{1}}^{1}$ by the Plücker embedding. Hence, the subspace $\mathcal{E}_{\omega, m^{1}}$ of $M_{m^{1}}^{(2)}$ can be regarded as a subspace of $\mathbb{P} \Lambda^{2} \mathcal{C}_{m^{1}}^{1}$, and (3.1) tells precisely that such a subspace coincides with $\mathcal{H}$ defined by (6.5).

We are now in position to generalize a result about classical MAEs [2, Theorem 3.7], to the context of $3^{\text {rd }}$ order MAEs.

Proposition 6.4. A characteristic direction for $\mathcal{E}_{\omega}$ is also strongly characteristic.

Proof. Let $H \in \mathbb{P C}_{m_{1}}^{1}$ be a characteristic direction for $\mathcal{E}_{\omega}$ at the point $m^{2}$. This means that $H \subset L_{m^{2}}$ and that the prolongation $H^{(1)}$ determined by $H$ is tangent to $\mathcal{E}_{\omega, m^{1}}$ at $m^{2}$. We need to prove that the whole $H^{(1)}$ is contained in $\mathcal{E}_{\omega, m^{1}}$.

To this end, recall that $H^{(1)} \subset M_{m^{1}}^{(2)}$ is a $1 \mathrm{D}$ affine subspace modeled over $S^{3}$ Ann $H$, passing through $L_{m^{2}}$ (see, e.g., the proof of Theorem 1 in [7]). By the above arguments, $H^{(1)}$ can be embedded into $\mathbb{P} \Lambda^{2} \mathcal{C}_{m^{1}}^{1}$ as well. Now we can compare $H^{(1)}$ and $\mathcal{H}$ : they are both linear, they pass through the same point $L_{m^{2}}$, where they are also tangent each other. Hence, $H^{(1)} \subset \mathcal{H}$.

Now Proposition 6.4 allows us to prove Theorem 3.3.

Proof of Theorem 3.3. Inclusion (6.3) is valid for any $3^{\text {rd }}$ order PDE. Conversely, if $m^{2} \in \mathcal{E}_{\mathcal{V}^{\mathcal{E}}}$, then there is a line $H \in \mathcal{V}^{\mathcal{E}}$, such that $L_{m^{2}} \supset H$. But $H$ is a strong characteristic line for $\mathcal{E}$ thanks to Proposition 6.4, so that all Lagrangian planes passing through $H$ and, in particular, $L_{m^{2}} \equiv m^{2}$ itself, belong to $\mathcal{E}$.

\subsection{Proof of Theorem 3.4}

As it was outlined in Section 3, Goursat-type MAEs are MAEs which correspond to decomposable forms, modulo a certain ideal. Before proving Theorem 3.4, we make rigorous this statement. To this end, we shall need the submodule of contact 2-forms

$$
\Theta=\left\{\omega \in \Lambda^{2} M^{(1)}|\omega|_{L_{m^{2}}}=0 \forall m^{2} \in M^{(2)}\right\} \subset \Lambda^{2} M^{(1)},
$$


and the corresponding projection

$$
\Lambda^{2} M^{(1)} \longrightarrow \frac{\Lambda^{2} M^{(1)}}{\Theta}
$$

Observe that the quotient bundle $\frac{\Lambda^{2} M^{(1)}}{\Theta}$ is canonically isomorphic to the rank-one bundle $\Lambda^{2} L^{*}$ over $M^{(1)}$. Hence, (6.7) can be thought of as $\left(\Lambda^{2} L^{*}\right)$-valued.

Remark 6.5. Two 2 -forms have the same projection (6.7) if and only if they differ by an element of $\Theta$.

Proposition 6.6. For a $3 D$ distribution $\mathcal{D} \subset \mathcal{C}^{1}$, it holds

$$
\mathcal{E}_{\mathcal{D}}=\mathcal{E}_{\omega} \Leftrightarrow \omega=\rho_{1} \wedge \rho_{2} \bmod \Theta
$$

Moreover, the $3 D$ sub-distribution $\mathcal{D} \subseteq \mathcal{C}^{1}$ is given by

$$
\mathcal{D}=\left.\left.\operatorname{ker} \rho_{1}\right|_{\mathcal{C}^{1}} \cap \operatorname{ker} \rho_{2}\right|_{\mathcal{C}^{1}}
$$

Proof. If $\operatorname{Ann} \mathcal{D}=\left\langle\rho_{1}, \rho_{2}\right\rangle \subset \mathcal{C}^{1 *}$ is the annihilator of $\mathcal{D}$ in $\mathcal{C}^{1}$, then $\mathcal{E}_{\mathcal{D}}$ can be written as $\mathcal{E}_{\widetilde{\rho_{1}} \wedge \widetilde{\rho_{2}}}$, where $\widetilde{\rho_{1}}, \widetilde{\rho_{2}} \in \Lambda^{1} M^{(1)}$ are extensions of $\rho_{1}, \rho_{2}$, respectively. The result follows from Remark 6.5.

Conversely, in light of (3.1) and (6.6), $\omega$ and $\rho_{1} \wedge \rho_{2}+\Theta$ give rise to the same equation, i.e.,

$$
\mathcal{E}_{\omega}=\mathcal{E}_{\rho_{1} \wedge \rho_{2}}
$$

It remains to be proved that the right-hand side of $(6.9)$ is of the form $\mathcal{E}_{\mathcal{D}}$. To this end, it suffices to define $\mathcal{D}$ as in (6.8),

$$
\mathcal{E}_{\mathcal{D}}=\widetilde{\mathcal{E}_{\left.\rho_{1}\right|_{\mathcal{C}^{1}}} \wedge \widetilde{\left.\rho_{2}\right|_{\mathcal{C}^{1}}}}
$$

and observe that the right-hand sides of (6.9) and (6.10) coincide since $\widetilde{\left.\rho_{i}\right|_{\mathcal{C}^{1}}}-\rho_{i} \in$ Ann $\mathcal{C}^{1} \subset \Theta$, $i=1,2$.

Before starting the proof of Theorem 3.4, we provide a meta-symplectic analog of the wellknown formula $\left.\operatorname{Ann} \mathcal{D}^{\perp}=\mathcal{D}\right\lrcorner \omega$.

Proposition 6.7. Let $\mathcal{D}_{1} \subseteq \mathcal{C}^{1}$ be a $3 D$ sub-distribution with $\operatorname{dim} \mathcal{D}_{1}^{v}=2$ and $\mathcal{E}=\mathcal{E}_{\mathcal{D}_{1}}$ the corresponding quasi-linear $3^{\text {rd }}$ order $M A E$. Then, if $\mathcal{V}_{\mathrm{II}}^{\mathcal{E}}=\mathbb{P D}_{2} \cup \mathbb{P} \mathcal{D}_{3}$,

$$
\operatorname{Ann} \mathcal{D}_{i}=\frac{\left.\mathcal{D}_{1}\right\lrcorner \Omega}{H_{i}} \subseteq \mathcal{C}^{1 *}, \quad i=2,3
$$

where $\mathrm{H}_{2}$ and $\mathrm{H}_{3}$ are the characteristic lines of $\mathcal{D}_{1}^{v}$.

Proof. Let $\mathcal{D}_{1}=\left\langle a D_{1}+b D_{2}, X_{1}, X_{2}\right\rangle$, with $X_{1}$ and $X_{2}$ as in (4.13). Then, the quasi-linear $3^{\text {rd }}$ order MAE determined by $\mathcal{D}_{1}$ is

$$
\mathcal{E}_{\mathcal{D}_{1}}=a p_{111}+\left(b-a\left(k_{1}+k_{2}\right)\right) p_{112}+\left(a k_{1} k_{2}-b\left(k_{1}+k_{2}\right)\right) p_{122}+b k_{1} k_{2} p_{222}
$$

In order to obtain the right-hand side of (6.11), compute first $\left.a D_{1}+b D_{2}\right\lrcorner \Omega=\left(a d p_{11}+b d p_{12}\right) \otimes$ $\partial_{p_{1}}+\left(a d p_{12}+b d p_{22}\right) \otimes \partial_{p_{2}}$, and factor it by $H_{i}$,

$$
\left.a D_{1}+b D_{2}\right\lrcorner \Omega=\left(a d p_{11}+\left(b-k_{i} a\right) d p_{12}-k_{i} b d p_{22}\right) \otimes \partial_{p_{1}} \quad \bmod H_{i} .
$$


Combining (6.12) above with (4.15), yields

$$
\frac{\left.\mathcal{D}_{1}\right\lrcorner \Omega}{H_{i}} \simeq\left\langle k_{j} d x^{1}+d x^{2}, a d p_{11}+\left(b-k_{i} a\right) d p_{12}-k_{i} b d p_{22}\right\rangle .
$$

Finally, the wedge product of the two 1-forms spanning the module (6.13) above, is the 2 -form

$$
\begin{aligned}
\omega= & a k_{j} d p_{11} \wedge d x^{1}+\left(b-k_{i} a\right) k_{j} d p_{12} \wedge d x^{1}-b k_{i} k_{j} d p_{22} \wedge d x^{1} \\
& +a d p_{11} \wedge d x^{2}+\left(b-k_{i} a\right) d p_{12} \wedge d x^{2}-k_{i} b d p_{22} \wedge d x^{2}
\end{aligned}
$$

and direct computations show that $\mathcal{E}_{\omega}=\mathcal{E}_{\mathcal{D}_{1}}$. The result follows from Proposition 6.6.

Now we turn back to Theorem 3.4, and deal separately with its two implications.

\subsubsection{Proof of the sufficient part of Theorem 3.4}

The sufficient part of Theorem 3.4 will be proved through Lemma 6.8 below.

Lemma 6.8. Let $\mathcal{E}=\{F=0\}$, where $F$ is given either by (3.16), or

$$
F=a p_{111}+b p_{112}+c p_{122}+d p_{222}+e
$$

where $a, b, c, d, e \in C^{\infty}\left(M^{(1)}\right)$. Then there exists a $3 D$ sub-distribution $\mathcal{D} \subseteq \mathcal{C}^{(1)}$ such that $\mathcal{V}^{\mathcal{E}}=\mathcal{V}_{\mathrm{I}}^{\mathcal{E}} \cup \mathcal{V}_{\mathrm{II}}^{\mathcal{E}}$, with $\mathcal{V}_{\mathrm{I}}^{\mathcal{E}}=\mathbb{P} \mathcal{D}$. Moreover, $\mathcal{E}=\mathcal{E}_{\mathcal{D}}$, i.e., according to (3.4), it is a $3^{\text {rd }}$ order $M A E$ of Goursat-type.

We recall that Lemma 6.8 is to be interpreted in fibre-wise perspective (see Section 4.5), i.e., as a collection of statements, each of which corresponds to the fixation of a point $m^{1} \in M^{(1)}$. But, since the formula (6.14) must correspond to an affine hyperplane in the space of the $p_{i j k}$ 's over $m^{1}$, then the vector $\left(a\left(m^{1}\right), b\left(m^{1}\right), c\left(m^{1}\right), d\left(m^{1}\right)\right) \in \mathbb{R}^{4}$ must be non-zero. This means that for any $m^{1} \in M^{(1)}$, there is always one of the functions $a, b, c, d$, which is non-zero at $m^{1}$.

Proof of Lemma 6.8. Let us begin with the non-linear case.

In order to verify that $\mathcal{E}=\mathcal{E}_{\mathcal{D}}$, it suffices to put

$$
\begin{aligned}
\mathcal{D}:= & \left\langle D_{1}+f_{111} \partial_{p_{11}}+f_{112} \partial_{p_{12}}+f_{122} \partial_{p_{22}}, D_{2}+f_{211} \partial_{p_{11}}+f_{212} \partial_{p_{12}}+f_{222} \partial_{p_{22}},\right. \\
& \left.R \partial_{p_{11}}+S \partial_{p_{12}}+T \partial_{p_{22}}\right\rangle,
\end{aligned}
$$

where $(R, S, T)=\boldsymbol{A}$ is the same appearing in (3.16), and observe that (see also (3.11))

$$
L_{m^{2}}=\left\langle D_{1}+p_{111} \partial_{p_{11}}+p_{112} \partial_{p_{12}}+p_{122} \partial_{p_{22}}, D_{2}+p_{112} \partial_{p_{11}}+p_{122} \partial_{p_{12}}+p_{222} \partial_{p_{22}}\right\rangle
$$

belongs to $\mathcal{E}_{\mathcal{D}}($ see $(3.4))$ if and only if the $5 \times 5$ determinant

$$
\operatorname{det}\left|\begin{array}{ccccc}
1 & 0 & p_{111} & p_{112} & p_{122} \\
0 & 1 & p_{112} & p_{122} & p_{222} \\
1 & 0 & f_{111} & f_{112} & f_{122} \\
0 & 1 & f_{211} & f_{212} & f_{222} \\
0 & 0 & R & S & T
\end{array}\right|=\operatorname{det}\left|\begin{array}{ccccc}
0 & 0 & p_{111}-f_{111} & p_{112}-f_{112} & p_{122}-f_{122} \\
0 & 0 & p_{112}-f_{211} & p_{122}-f_{212} & p_{222}-f_{222} \\
1 & 0 & f_{111} & f_{112} & f_{122} \\
0 & 1 & f_{211} & f_{212} & f_{222} \\
0 & 0 & R & S & T
\end{array}\right|=-F
$$

is zero.

We now prove that $\mathcal{V}_{\mathrm{I}}^{\mathcal{E}}=\mathbb{P} \mathcal{D}$ in the case the coefficient of $p_{111}$ of equation (3.16) is not zero. The other cases are formally analog. So, let us solve equation (3.16) with respect to $p_{111}$ and 
take the remaining coordinates $p_{112}, p_{122}, p_{222}$ as local coordinates on $\mathcal{E}$. Thus $\operatorname{Smbl} F(\operatorname{see}(5.6))$ is, up to a factor, equal to

$$
\begin{aligned}
& \left(\left(S\left(p_{222}-f_{222}\right)-T\left(p_{122}-f_{212}\right)\right) \xi_{1}+\left(T\left(p_{112}-f_{112}\right)-S\left(p_{122}-f_{122}\right)\right) \xi_{2}\right) \\
& \quad \times\left(\left(T\left(p_{122}-f_{212}\right)-S\left(p_{222}-f_{222}\right)\right) \xi_{1}^{2}+\left(R\left(p_{222}-f_{222}\right)-T\left(p_{112}-f_{211}\right)\right) \xi_{1} \xi_{2}\right. \\
& \left.\quad+\left(S\left(p_{112}-f_{211}\right)-R\left(p_{122}-f_{212}\right)\right) \xi_{2}^{2}\right)
\end{aligned}
$$

so that (see also Section 5.3)

$$
\begin{aligned}
\mathcal{V}_{\mathrm{I}}^{\mathcal{E}}= & \left\{\left(\left(S\left(p_{222}-f_{222}\right)-T\left(p_{122}-f_{212}\right)\right) D_{1}\right.\right. \\
& \left.\left.+\left(T\left(p_{112}-f_{112}\right)-S\left(p_{122}-f_{122}\right)\right) D_{2}\right)_{m^{2}} \mid m^{2} \in \mathcal{E}\right\} .
\end{aligned}
$$

A direct computation shows that

$$
\begin{aligned}
\mathcal{V}_{\mathrm{I}}^{\mathcal{E}}= & \left\{\left(T\left(p_{122}-f_{212}\right)-S\left(p_{222}-f_{222}\right)\right)\left(D_{1}+f_{111} \partial_{p_{11}}+f_{112} \partial_{p_{12}}+f_{122} \partial_{p_{22}}\right)\right. \\
& +\left(S\left(p_{122}-f_{122}\right)-T\left(p_{112}-f_{112}\right)\right)\left(D_{2}+f_{211} \partial_{p_{11}}+f_{212} \partial_{p_{12}}+f_{222} \partial_{p_{22}}\right) \\
& +\left(-p_{112} p_{222}+p_{112} f_{222}+f_{112} p_{222}-f_{112} f_{222}+p_{122}^{2}-p_{122} f_{212}-f_{122} p_{122}+f_{122} f_{212}\right) \\
& \left.\times\left(R \partial_{p_{11}}+S \partial_{p_{12}}+T \partial_{p_{22}}\right) \mid p_{112}, p_{122}, p_{222} \in \mathbb{R}\right\}
\end{aligned}
$$

so that $\mathcal{V}_{\mathrm{I}}^{\mathcal{E}}$ turns out to be the $3 \mathrm{D}$ linear space (6.15).

Let us now pass to the quasi-linear case.

One of the coefficients of the third derivatives of (6.14) must be non-zero. Assume that $a \neq 0$; the remaining cases can be treated similarly. Again, we solve equation (6.14) with respect to $p_{111}$, so that $p_{112}, p_{122}, p_{222}$ become local coordinates on it, and

$$
\operatorname{Smbl} F=a \xi_{1}^{3}+b \xi_{1}^{2} \xi_{2}+c \xi_{1} \xi_{2}^{2}+d \xi_{2}^{3}=\left(k \xi_{1}+h \xi_{2}\right) q\left(\xi_{1}, \xi_{2}\right)
$$

where $q\left(\xi_{1}, \xi_{2}\right)$ is a homogeneous quadratic function in $\xi_{1}$ and $\xi_{2}$. Thus, we have that (see again Section 5.3).

$$
\mathcal{V}_{\mathrm{I}}^{\mathcal{E}}=\left\langle\left(k D_{1}+h D_{2}\right)_{m^{2}} \mid m^{2} \in \mathcal{E}\right\rangle \text {. }
$$

Let us assume $d=0$. In order to verify that $\mathcal{E}=\mathcal{E}_{\mathcal{D}}$, it is enough to put

$$
\mathcal{D}:=\left\langle D_{1}-\frac{e}{a} \partial_{p_{11}},-\frac{b}{a} \partial_{p_{11}}+\partial_{p_{12}},-\frac{c}{a} \partial_{p_{11}}+\partial_{p_{22}}\right\rangle \text {. }
$$

To prove that $\mathcal{V}_{\mathrm{I}}^{\mathcal{E}}=\mathbb{P D}$, observe that the symbol (6.18) contains the linear factor $\xi_{1}$, and

$$
\begin{aligned}
\mathcal{V}_{\mathrm{I}}^{\mathcal{E}} & =\left\langle\left. D_{1}\right|_{m^{2}}\right\rangle_{m^{2} \in \mathcal{E}} \\
& =\left\{D_{1}-\frac{e}{a} \partial_{p_{11}}+p_{112}\left(-\frac{b}{a} \partial_{p_{11}}+\partial_{p_{12}}\right)+p_{122}\left(-\frac{c}{a} \partial_{p_{11}}+\partial_{p_{22}}\right) \mid p_{112}, p_{122} \in \mathbb{R}\right\}
\end{aligned}
$$

is the projectivization of (6.20).

Finally assume $d \neq 0$.

Observe that the lines in $\mathcal{V}_{\mathrm{I}}^{\mathcal{E}}$, which, in view of (6.19), are generated by

$$
\begin{aligned}
k D_{1} & +h D_{2}-\frac{k e}{a} \partial_{p_{11}}+p_{112}\left(\left(-\frac{k b}{a}+h\right) \partial_{p_{11}}+k \partial_{p_{12}}\right) \\
& +p_{122}\left(-\frac{k c}{a} \partial_{p_{11}}+h \partial_{p_{12}}+k \partial_{p_{22}}\right)+p_{222}\left(-\frac{k d}{a} \partial_{p_{11}}+h \partial_{p_{22}}\right)
\end{aligned}
$$


$p_{112}, p_{122}, p_{222} \in \mathbb{R}$, fill a $3 \mathrm{D}$ linear space since $\frac{\xi_{1}}{\xi_{2}}=-\frac{h}{k}$ is a solution to (6.18), i.e.,

$$
-a \frac{h^{3}}{k^{3}}+b \frac{h^{2}}{k^{2}}-c \frac{h}{k}+d=0 .
$$

So, if we put

$$
\mathcal{D}:=\left\langle D_{1}+\frac{h}{k} D_{2}-\frac{e}{a} \partial_{p_{11}},\left(-\frac{b}{a}+\frac{h}{k}\right) \partial_{p_{11}}+\partial_{p_{12}}, \frac{k}{h} \frac{d}{a} \partial_{p_{11}}-\partial_{p_{22}}\right\rangle
$$

with $h, k \neq 0$ such that $(6.21)$ is satisfied, we obtain $\mathcal{E}=\mathcal{E}_{\mathcal{D}}$ and $\mathcal{V}_{\mathrm{I}}^{\mathcal{E}}=\mathbb{P} \mathcal{D}$.

\subsubsection{Proof of the necessary part of Theorem 3.4}

Being $\mathcal{E}_{m^{1}}$ a closed submanifold of codimension 1, in the neighborhood of any point $m^{2} \in \mathcal{E}_{m^{1}}$, we can always present $\mathcal{E}$ in the form $\mathcal{E}=\{F=0\}$, with $F:=p_{i j k}-G$, with $G$ not depending on $p_{i j k}$, for some $(i, j, k)$ which we shall assume equal to $(1,1,1)$, since the other cases are formally analog. Then the symbol of $F$ at $m^{1}$ is

$$
\mathrm{Smbl}_{m^{1}} F=\xi_{1}^{3}-G_{p_{112}} \xi_{1}^{2} \xi_{2}-G_{p_{122}} \xi_{1} \xi_{2}^{2}-G_{p_{222}} \xi_{2}^{3}
$$

The right-hand side of (6.22) is a $3^{\text {rd }}$ order homogeneous polynomial with unit leading coefficient: hence, there exist unique $\beta, A, B$ such that $\operatorname{Smbl}_{m^{1}} F=\left(\xi_{1}+\beta \xi_{2}\right)\left(\xi_{1}^{2}+A \xi_{1} \xi_{2}+B \xi_{2}^{2}\right)$. Following the general procedure (see also formula (5.9) and Definition 5.7), to construct the characteristic cone $\mathcal{V}^{\mathcal{E}}$, one easily sees that $\mathcal{V}^{\mathcal{E}}$ contains the following 3-parametric family of lines:

$$
\begin{aligned}
\mathcal{V}_{\mathrm{I}}^{\mathcal{E}}:=\{ & D_{1}+\beta D_{2}+\left(G+\beta p_{112}\right) \partial_{p_{11}}+\left(p_{112}+\beta p_{122}\right) \partial_{p_{12}} \\
& \left.+\left(p_{122}+\beta p_{222}\right) \partial_{p_{22}} \mid p_{112}, p_{122}, p_{222} \in \mathbb{R}\right\} .
\end{aligned}
$$

Observe that, with the same notation as (1.8), the direction $v=\left(v^{1}, v^{2}\right)$ belongs to $\mathcal{V}_{\mathrm{I}}^{\mathcal{E}}$ if and only if there exists $m^{2} \in \mathcal{E}_{m^{1}}$ such $v^{1}+\beta\left(m^{2}\right) v^{2}=0$ (see also Remark 4.7).

Suppose now that there exists a 3D sub-distribution $\mathcal{D} \subset \mathcal{C}^{1}$ such that

$$
\mathcal{V}_{\mathrm{I}}^{\mathcal{E}}=\mathbb{P} \mathcal{D} .
$$

Since $\mathcal{D}$ has codimension 2 in $\mathcal{C}^{1}$, condition (6.23) can be dualized as follows: there are two independent forms $\rho_{1}, \rho_{2} \in \mathcal{C}^{1 *}$,

$$
\begin{aligned}
& \rho_{1}=k_{1} d x^{1}+k_{2} d x^{2}+k^{11} d p_{11}+k^{12} d p_{12}+k^{22} d p_{22}, \\
& \rho_{2}=h_{1} d x^{1}+h_{2} d x^{2}+h^{11} d p_{11}+h^{12} d p_{12}+h^{22} d p_{22},
\end{aligned}
$$

such that $\operatorname{Ann} \mathcal{D}=\left\langle\rho_{1}, \rho_{2}\right\rangle$, i.e., $\mathcal{D}=\operatorname{ker} \rho_{1} \cap \operatorname{ker} \rho_{2}$. In other words, (6.23) holds true if and only if $\rho_{1}(v)=\rho_{2}(v)=0$ for all $v \in \mathcal{V}_{\text {I }}^{\mathcal{E}}$, i.e.,

$$
\begin{aligned}
& k_{1}+k^{12} p_{112}+k^{22} p_{122}+\left(k_{2}+k^{11} p_{112}+k^{12} p_{122}+k^{22} p_{222}\right) \beta+k^{11} G=0, \\
& h_{1}+h^{12} p_{112}+h^{22} p_{122}+\left(h_{2}+h^{11} p_{112}+h^{12} p_{122}+h^{22} p_{222}\right) \beta+h^{11} G=0,
\end{aligned}
$$

identically in $p_{112}, p_{122}, p_{222}$, where the $2 \times 5$ matrix

$$
\left|\begin{array}{lllll}
k_{1} & k_{2} & k^{11} & k^{12} & k^{22} \\
h_{1} & h_{2} & h^{11} & h^{12} & h^{22}
\end{array}\right|
$$

of functions on $M^{(1)}$ has rank two everywhere. 
Now (6.24), (6.25) must be regarded as linear system of two equations in the unknowns $\beta$ and $G$. Its discriminant is easily computed, $\Delta:=\left(h^{11} k^{22}-k^{11} h^{22}\right) p_{222}+\left(h^{11} k^{12}-k^{11} h^{12}\right) p_{122}+$ $h^{11} k_{2}-k^{11} h_{2}$, and $\Delta$ is a polynomial in $p_{122}, p_{222}$.

Suppose $\Delta\left(m^{2}\right)=0$, and interpret (6.24), (6.25) as a linear system in the variables $\beta$ and $G$. The condition $\Delta\left(m^{2}\right)=0$, together with the compatibility conditions of such a system, implies that the matrix (6.26) is of rank $1 \mathrm{in} m^{1}$. This contradicts the hypothesis that $\operatorname{dim} \mathcal{D}_{m^{1}}=3$, so that $\Delta$ must be everywhere non-zero.

Then, in the points with $\Delta\left(p_{122}, p_{222}\right) \neq 0$ one can find $\Delta G$ as a polynomial expression of $p_{112}, p_{122}, p_{222}$, whose coefficients turn out to be minors of the matrix (6.26). In particular, $p_{111}-G$ can be singled out from the so-obtained expression, namely

$$
\begin{aligned}
-\Delta\left(p_{111}-G\right)= & -h_{1} k_{2}+k_{1} h_{2}-\left(h^{11} k_{2}-k^{11} h_{2}\right) p_{111} \\
& -\left(k^{11} h_{1}-h^{11} k_{1}+k_{2} h^{12}-k^{12} h_{2}\right) p_{112} \\
& -\left(k_{2} h^{22}+k^{12} h_{1}-k^{22} h_{2}-k_{1} h^{12}\right) p_{122}-\left(k^{22} h_{1}-k_{1} h^{22}\right) p_{222} \\
& +\left(k^{12} h^{22}-h^{12} k^{22}\right)\left(p_{112} p_{222}-p_{122}^{2}\right) \\
& +\left(k^{22} h^{11}-k^{11} h^{22}\right)\left(-p_{111} p_{222}+p_{112} p_{122}\right) \\
& +\left(k^{11} h^{12}-h^{11} k^{12}\right)\left(p_{111} p_{122}-p_{112}^{2}\right) .
\end{aligned}
$$

We need to show that (6.27) is satisfied if and only if there is a nowhere zero factor $\lambda$ such that

$$
F=\lambda\left(p_{111}-G\right),
$$

where $F$ is given by (3.16), i.e., equation (6.28) needs to be solved with respect to $f_{i j k}, R, S, T$. By equating the coefficients of $p_{111}$, one obtains

$$
\lambda=T\left(p_{122}-f_{212}\right)-S\left(p_{222}-f_{222}\right),
$$

and replacing (6.29) into (6.28) yields

$$
F-\left(T\left(p_{122}-f_{212}\right)-S\left(p_{222}-f_{222}\right)\right)\left(p_{111}-G\right)=0 .
$$

Direct computations show that the left-hand side of (6.30) is a rational function whose numerator is a $2^{\text {nd }}$ order polynomial in $p_{112}, p_{122}, p_{222}$. From the vanishing of this polynomial, one can express $f_{i j k}, R, S, T$ as functions of the entries of the matrix (6.26).

\subsection{Proof of Theorem 3.5}

It will be accomplished in steps. As a preparatory result, we show that a 3D sub-distribution of $\mathcal{C}^{1}$ with $2 \mathrm{D}$ vertical part determines a quasi-linear $3^{\text {rd }}$ order MAE (Lemma 6.9 below). The converse statement, i.e., that the characteristic cone of a generic quasi-linear $3^{\text {rd }}$ order PDE possesses a linear sheet determined by a $3 \mathrm{D}$ sub-distribution $\mathcal{D} \subseteq \mathcal{C}^{1}$ with $2 \mathrm{D}$ vertical part, has been proved by Lemma 6.8 above. Then we pass to generic $3 \mathrm{D}$ sub-distributions of $\mathcal{C}^{1}$, and derive the expression of the corresponding equation $\mathcal{E}_{\mathcal{D}}$ (Lemma 6.11). As a consequence of these results (Corollary 6.14), we prove the initial statement of Theorem 3.5.

The next Sections 6.4.1, 6.4.2, 6.4.3 are devoted to the specific proofs of the items 1, 2, 3 of Theorem 3.5, respectively.

Besides the proof of Theorem 3.5 itself, a few interesting byproducts will be pointed out. For instance, Corollary 6.14 means that for quasi-linear $3^{\text {rd }}$ order MAEs, all characteristic lines are strong, generalizing an analogous result for classical MAEs (see [2, Theorem 3.7]). Also Corollary 6.17 is a non-trivial and unexpected generalization of a phenomenon firstly observed in the classical case (see [2, Theorem 1.1]). 
Lemma 6.9. Let $\mathcal{D}_{1}=\left\langle h_{1}, \mathcal{D}_{1}^{v}\right\rangle$, with

$$
\begin{aligned}
& h_{1}=a D_{1}+b D_{2}+f_{11} \partial_{p_{11}}+f_{12} \partial_{p_{12}}+f_{22} \partial_{p_{22}}, \\
& \mathcal{D}_{1}^{v}=\left\langle R_{i} \partial_{p_{11}}+S_{i} \partial_{p_{12}}+T_{i} \partial_{p_{22}} \mid i=1,2\right\rangle .
\end{aligned}
$$

Then $\mathcal{E}_{\mathcal{D}_{1}}$ is of the form (3.15) with

$$
\begin{aligned}
& \boldsymbol{A}=0, \\
& C=\operatorname{det}\left|\begin{array}{lll}
f_{11} & f_{12} & f_{22} \\
R_{1} & S_{1} & T_{1} \\
R_{2} & S_{2} & T_{2}
\end{array}\right|, \\
& \boldsymbol{B}=\left(-a \operatorname{det}\left|\begin{array}{ll}
S_{1} & T_{1} \\
S_{2} & T_{2}
\end{array}\right|,-b \operatorname{det}\left|\begin{array}{ll}
S_{1} & T_{1} \\
S_{2} & T_{2}
\end{array}\right|+a \operatorname{det}\left|\begin{array}{ll}
R_{1} & T_{1} \\
R_{2} & T_{2}
\end{array}\right|,\right. \\
&\left.\quad b \operatorname{det}\left|\begin{array}{ll}
R_{1} & T_{1} \\
R_{2} & T_{2}
\end{array}\right|-a \operatorname{det}\left|\begin{array}{ll}
R_{1} & S_{1} \\
R_{2} & S_{2}
\end{array}\right|,-b \operatorname{det}\left|\begin{array}{ll}
R_{1} & S_{1} \\
R_{2} & S_{2}
\end{array}\right|\right) .
\end{aligned}
$$

Proof. Just observe that

$$
L_{m^{2}}=\left\langle D_{1}+p_{111} \partial_{p_{11}}+p_{112} \partial_{p_{12}}+p_{122} \partial_{p_{22}}, D_{2}+p_{112} \partial_{p_{11}}+p_{122} \partial_{p_{12}}+p_{222} \partial_{p_{22}}\right\rangle
$$

belongs to $\mathcal{E}_{\mathcal{D}_{1}}$ if and only if the determinant of the $5 \times 5$ matrix

$$
\left|\begin{array}{ccccc}
a & b & f_{11} & f_{12} & f_{22} \\
0 & 0 & R_{1} & S_{1} & T_{1} \\
0 & 0 & R_{2} & S_{2} & T_{2} \\
1 & 0 & p_{111} & p_{112} & p_{122} \\
0 & 1 & p_{112} & p_{122} & p_{222}
\end{array}\right|
$$

is zero (see also (3.11) and (3.4)). Standard matrix manipulations reveal that the determinant of (6.34) coincides in turn with (3.15) under conditions (6.31), (6.32) and (6.33).

Remark 6.10. Since $\operatorname{dim} \operatorname{Gr}(3,5)=6$, it takes 6 parameters from $C^{\infty}\left(M^{(1)}\right)$ to identify a 3D sub-distribution $\mathcal{D} \subset \mathcal{C}^{1}$ and, hence, an equation of the form $\mathcal{E}_{\mathcal{D}}$.

Above Remark 6.10 shows that the description (3.16) of the $\mathcal{E}_{\mathcal{D}}$ 's has some redundancies, and Lemma 6.13) below is a way to refine it.

Lemma 6.11. The local form of a generic $\mathcal{E}_{\mathcal{D}}$ with $\mathcal{D} \in \operatorname{Gr}\left(3, \mathcal{C}^{1}\right)$, in the case $\operatorname{dim} \mathcal{D}^{v}=1$, is given by (3.16).

Proof. A distribution $\mathcal{D} \in \operatorname{Gr}\left(3, \mathcal{C}^{1}\right)$ with $\operatorname{dim} \mathcal{D}^{v}=1$ is locally given by

$$
\begin{aligned}
\mathcal{D}=\langle & D_{1}+f_{111} \partial_{p_{11}}+f_{112} \partial_{p_{12}}+f_{122} \partial_{p_{22}}, D_{2}+f_{211} \partial_{p_{11}}+f_{212} \partial_{p_{12}}+f_{222} \partial_{p_{22}}, \\
& \left.R \partial_{p_{11}}+S \partial_{p_{12}}+T \partial_{p_{22}}\right\rangle,
\end{aligned}
$$

where $f_{i j k}, R, S, T$ are $C^{\infty}$ functions (defined in some neighborhood of $M^{(1)}$ ). Now it is clear that a Lagrangian plane

$$
\left\langle D_{1}+p_{111} \partial_{p_{11}}+p_{112} \partial_{p_{12}}+p_{122} \partial_{p_{22}}, D_{2}+p_{112} \partial_{p_{11}}+p_{122} \partial_{p_{12}}+p_{222} \partial_{p_{22}}\right\rangle
$$

non-trivially intersects $\mathcal{D}$ iff

$$
\operatorname{det}\left(\begin{array}{ccccc}
1 & 0 & p_{111} & p_{112} & p_{122} \\
0 & 1 & p_{112} & p_{122} & p_{222} \\
1 & 0 & f_{111} & f_{112} & f_{122} \\
0 & 1 & f_{211} & f_{212} & f_{222} \\
0 & 0 & R & S & T
\end{array}\right)=\left(\begin{array}{ccc}
p_{111}-f_{111} & p_{112}-f_{112} & p_{122}-f_{122} \\
p_{112}-f_{211} & p_{122}-f_{212} & p_{222}-f_{222} \\
R & S & T
\end{array}\right)=0
$$

that is equal to (3.16) with $\boldsymbol{A}=(R, S, T)$. 
Now we discuss the possibility of reducing the number of redundant parameters in (3.16) (see Remark 6.10). In turn, this is linked to the natural question whether there exists a Lagrangian horizontal part $\mathcal{H}$ of $\mathcal{D} \in \operatorname{Gr}\left(3, \mathcal{C}^{1}\right)$ in the case that $\operatorname{dim} \mathcal{D}^{v}=1$, i.e., the existence of a splitting $\mathcal{D}=\mathcal{H} \oplus \mathcal{D}^{v}$, with Lagrangian $\mathcal{H}$.

Lemma 6.12. Let $\mathcal{D} \subset \mathcal{C}^{1}$ be a $3 D$ distribution such that $\operatorname{dim} \mathcal{D}^{v}=1$. If there exists a Lagrangian horizontal part $\mathcal{H}$ of $\mathcal{D}$, then $\mathcal{E}_{\mathcal{D}}$ can be put in the form $\mathcal{E}=\{F=0\}$, where $F$ is given by (3.16) with $f_{112}=f_{211}$ and $f_{122}=f_{212}$.

Proof. It follows easily taking into account the computations of Lemma 6.11.

Lemma 6.13. Let $\mathcal{D} \subset \mathcal{C}^{1}$ be a $3 D$ distribution such that $\operatorname{dim} \mathcal{D}^{v}=1$. Let $m^{1} \in M^{(1)}$. If $\operatorname{rank}\left(\mathcal{D}_{m^{1}}^{v}\right) \neq 1$ (see Definition 4.6 concerning rank-one lines), then $\mathcal{E}_{\mathcal{D}}$ can be put, in a neighborhood of $m^{1}$, in the form $\mathcal{E}=\{F=0\}$, where $F$ is given by (3.16) with $f_{112}=f_{211}$ and $f_{122}=f_{212}$.

Proof. Let the vertical part $\mathcal{D}^{v}$ of $\mathcal{D}$ be spanned by

$$
\mathcal{V}=R \partial_{p_{11}}+S \partial_{p_{12}}+T \partial_{p_{22}}
$$

where $R, S, T \in C^{\infty}\left(M^{(1)}\right)$. Let

$$
\mathcal{D}=\left\langle D_{1}+b_{111} \partial_{p_{11}}+b_{112} \partial_{p_{12}}+b_{122} \partial_{p_{22}}, D_{2}+b_{211} \partial_{p_{11}}+b_{212} \partial_{p_{12}}+b_{222} \partial_{p_{22}}, \mathcal{V}\right\rangle,
$$

with $b_{i j k} \in C^{\infty}\left(M^{(1)}\right)$. For any $\alpha, \beta \in C^{\infty}\left(M^{(1)}\right)$ we have that

$$
\begin{aligned}
\mathcal{D}=\langle & D_{1}+b_{111} \partial_{p_{11}}+b_{112} \partial_{p_{12}}+b_{122} \partial_{p_{22}}+\alpha \mathcal{V}, \\
& \left.D_{2}+b_{211} \partial_{p_{11}}+b_{212} \partial_{p_{12}}+b_{222} \partial_{p_{22}}+\beta \mathcal{V}, \mathcal{V}\right\rangle .
\end{aligned}
$$

If $\operatorname{rank}\left(\mathcal{V}_{m^{1}}\right) \neq 1$, then $\alpha$ and $\beta$ can be chosen in such a way that, in a neighborhood of $m^{1}$,

$$
b_{112}+\alpha S=b_{211}+\beta R, \quad b_{122}+\alpha T=b_{212}+\beta S .
$$

In fact system (6.36) is always compatible for any $R, S, T, b_{112}, b_{211}, b_{122}, b_{212} \operatorname{since} \operatorname{rank}\left(\mathcal{V}_{m^{1}}\right) \neq 1$ iff $R T-S^{2} \neq 0$ at $m^{1}$. To conclude this part of the lemma it is enough to set

$$
f_{111}=b_{111}, \quad f_{112}=b_{112}+\alpha S, \quad f_{122}=b_{122}+\alpha T, \quad f_{222}=b_{222}
$$

and the corresponding $\mathcal{E}_{\mathcal{D}}$, with $\mathcal{D}$ given by (6.35), is precisely described by $(3.16)$ with $f_{112}=$ $f_{211}$ and $f_{122}=f_{212}$.

Corollary 6.14. Let $\mathcal{E}$ be an equation with $\mathcal{V}_{\mathrm{I}}^{\mathcal{E}}=\mathbb{P} \mathcal{D}_{1}$. Then $\mathcal{E}=\mathcal{E}_{\mathcal{D}_{1}}$.

Proof. Observe that any $L_{m^{2}}$, with $m^{2} \in \mathcal{E}$, always contains a characteristic line $H$ corresponding to a fixed linear factor of the symbol, i.e., an element $H$ belonging to $\mathcal{V}_{\mathrm{I}}^{\mathcal{E}}$ (see Section 5.3). In other words, inclusion (6.4) can be made more precise:

$$
\mathcal{E}_{\mathcal{V}_{\mathrm{I}}^{\mathcal{E}}} \supseteq \mathcal{E}
$$

It remains to prove that the inverse of inclusion (6.37) is valid when $\mathcal{V}_{\mathrm{I}}^{\mathcal{E}}=\mathbb{P} \mathcal{D}_{1}$. Indeed, Lemma 6.9 and Lemma 6.11 together guarantee that the left-hand side of (6.37) is a closed submanifold (possibly with singularities, see Section 4.5) of codimension one. But the right-hand side of (6.37) is a closed submanifold (again with possible singularities) of the same dimension of the left-hand side. Hence, the two of them must coincide as well. 


\subsubsection{Proof of the statement 1 of Theorem 3.5}

The proof of its first claim is contained in the next corollary.

Corollary 6.15. A $3^{\text {rd }}$ order $M A E \mathcal{E}_{\mathcal{D}}$ is quasi-linear if and only $\operatorname{dim} \mathcal{D}^{v}=2$.

Proof. A direct consequence of Lemmas 6.8, 6.9 and 6.11.

The remainder of statement 1 is concerned with the "other component" $\mathcal{V}_{\text {II }}^{\mathcal{E}}$ of $\mathcal{V}^{\mathcal{E}}$, i.e., the one associated with the quadratic factor of Ann $V \mathcal{E}$ (see Section 5.3). Recall that $\mathcal{V}_{\text {II }}^{\mathcal{E}}$ might be empty, in which case there is nothing to prove. At the far end, there is the case when $\mathcal{V}_{\text {II }}^{\mathcal{E}}$ is, in its turn, decomposable, i.e., it consists of two linear sheets, which is dealt with by Lemma 6.16 below.

Lemma 6.16. Let $\mathcal{E}=\{F=0\}$ be a $3^{\text {rd }}$ order quasi-linear PDE such that $\mathrm{Smbl} F$ is completely decomposable, i.e.,

$$
\text { Smbl } F=\left(\xi+k_{1} \eta\right) \odot\left(\xi+k_{2} \eta\right) \odot\left(\xi+k_{3} \eta\right) .
$$

If

$$
\mathcal{D}_{i}:=\left\langle\left. D_{1}\right|_{m^{2}}+\left.k_{i} D_{2}\right|_{m^{2}} \mid m^{2} \in \mathcal{E}_{m^{1}}\right\rangle
$$

is the $3 D$ sub-distribution of $\mathcal{C}^{1}$ corresponding ${ }^{11}$ to the $i^{\text {th }}$ linear factor of (6.38) and

$$
\overline{\mathcal{D}}=\left\langle\bar{h}, \overline{\mathcal{D}}^{v}\right\rangle \subseteq \mathcal{C}^{1}
$$

is a generic $3 D$ sub-distribution of $\mathcal{C}^{1}$ with $\operatorname{dim} \overline{\mathcal{D}}^{v}=2$, then $\overline{\mathcal{D}}$ satisfies condition (3.6) if and only if $\overline{\mathcal{D}}=\mathcal{D}_{j}$ with $j \neq i$.

Proof. To begin with, (6.38) dictates some restrictions on $F$, which must be of the form

$$
F=p_{111}+\left(k_{1}+k_{2}+k_{3}\right) p_{112}+\left(k_{1} k_{2}+k_{1} k_{3}+k_{2} k_{3}\right) p_{122}+k_{1} k_{2} k_{3} p_{222}+c .
$$

We begin with the "homogeneous" case, i.e., we assume $c=0$ since, as we shall see at the end of the proof, the general case can be easily brought back to this one.

Equating (6.41) to zero allows to express $p_{111}$ as a linear combination of $p_{112}, p_{122}$, and $p_{222}$, i.e., to identify $\mathcal{E}_{m^{1}}$ with $\mathbb{R}^{3} \equiv\left\{\left(p_{112}, p_{122}, p_{222}\right)\right\}$. In turn, this makes it possible to parametrize the space of vertical elements of (6.39) by three real parameters, viz.,

$$
\begin{aligned}
\mathcal{D}_{i}^{v}=\langle & -\left(\left(k_{1}+k_{2}+k_{3}-k_{i}\right) p_{112}+\left(k_{1} k_{2}+k_{1} k_{3}+k_{2} k_{3}\right) p_{122}+k_{1} k_{2} k_{3} p_{222}\right) \partial_{p_{11}} \\
& +\left(p_{112}+k_{i} p_{122}\right) \partial_{p_{12}}+\left(p_{122}+k_{i} p_{222}\right) \partial_{p_{22}}\left|p_{112}, p_{122}, p_{222} \in \mathbb{R}\right\rangle .
\end{aligned}
$$

It is worth observing that, in compliance with Lemma 6.8 , the dimension of $\mathcal{D}_{i}^{v}$, which equals the rank of the $3 \times 3$ matrix

$$
M_{i}=\left|\begin{array}{ccc}
-k_{1}-k_{2}-k_{3}+k_{i} & -k_{1} k_{2}-k_{1} k_{3}-k_{2} k_{3} & -k_{1} k_{2} k_{3} \\
1 & k_{i} & 0 \\
0 & 1 & k_{i}
\end{array}\right|
$$

is 2: indeed, $\operatorname{det} M_{i}=-\left(k_{1}-k_{i}\right)\left(k_{2}-k_{i}\right)\left(k_{3}-k_{i}\right)$ vanishes for $i=1,2,3$.

In order to find a basis for (6.42), regard the matrix (6.43) as a (rank-two) homomorphism $M_{i}: \mathbb{R}^{3} \longrightarrow V=\left\langle\partial_{p_{11}}, \partial_{p_{12}}, \partial_{p_{22}}\right\rangle$ and compute its kernel:

$$
\operatorname{ker} M_{i}=\left\langle k_{i}^{2},-k_{i}, 1\right\rangle .
$$

\footnotetext{
${ }^{11}$ See also, on this concern, the construction of $\mathcal{V}_{\mathrm{I}}^{\mathcal{E}}$ in Section 5.3.
} 
Then, independently on $i$ (and on the value of $k_{i}$ as well), $\mathbb{R}^{3}=\langle(1,0,0),(0,1,0)\rangle \oplus \operatorname{ker} M_{i}$, and $\mathcal{D}_{i}^{v}=\left\langle M_{i} \cdot(1,0,0), M_{i} \cdot(0,1,0)\right\rangle$ is the sought-for basis. In other words, instead of $(6.39)$, we shall work with the handier description $\mathcal{D}_{i}=\left\langle h_{i}, \mathcal{D}_{i}^{v}\right\rangle$, where

$$
\begin{aligned}
& h_{i}=D_{1}+k_{i} D_{2}, \\
& \mathcal{D}_{i}^{v}=\left\langle-\left(k_{1}+k_{2}+k_{3}-k_{i}\right) \partial_{p_{11}}+\partial_{p_{12}},-\left(k_{1} k_{2}+k_{1} k_{3}+k_{2} k_{3}\right) \partial_{p_{11}}+k_{i} \partial_{p_{12}}+\partial_{p_{22}}\right\rangle .
\end{aligned}
$$

Concerning (6.40), introduce similar descriptions of its horizontal and vertical part:

$$
\bar{h}=a D_{1}+b D_{2}, \quad \overline{\mathcal{D}}^{v}=\left\langle r_{1} \partial_{p_{11}}+s_{1} \partial_{p_{12}}+t_{1} \partial_{p_{22}}, r_{2} \partial_{p_{11}}+s_{2} \partial_{p_{12}}+t_{2} \partial_{p_{22}}\right\rangle .
$$

This concludes the preliminary part of the proof. Now impose condition (3.6):

$$
\Omega\left(h_{i}, \overline{\mathcal{D}}^{v}\right)=\Omega\left(\bar{h}, \mathcal{D}_{i}^{v}\right)=1 \mathrm{D} \text { subspace. }
$$

Observe that (6.46) consists, in fact, of two requirements, which are going to be dealt with separately.

The first one corresponds to the one-dimensionality of the subspace

$$
\begin{aligned}
\Omega\left(h_{i}, \overline{\mathcal{D}}^{v}\right) & =\left\langle r_{1} \partial_{p_{1}}+s_{1} \partial_{p_{2}}+k_{i}\left(s_{1} \partial_{p_{1}}+t_{1} \partial_{p_{2}}\right), r_{2} \partial_{p_{1}}+s_{2} \partial_{p_{2}}+k_{i}\left(s_{2} \partial_{p_{1}}+t_{2} \partial_{p_{2}}\right)\right\rangle \\
& =\left\langle\left(r_{1}+k_{i} s_{1}\right) \partial_{p_{1}}+\left(s_{1}+k_{i} t_{1}\right) \partial_{p_{2}},\left(r_{2}+k_{i} s_{2}\right) \partial_{p_{1}}+\left(s_{2}+k_{i} t_{2}\right) \partial_{p_{2}}\right\rangle,
\end{aligned}
$$

i.e., to the equation

$$
\operatorname{det}\left|\begin{array}{ll}
r_{1}+k_{i} s_{1} & s_{1}+k_{i} t_{1} \\
r_{2}+k_{i} s_{2} & s_{2}+k_{i} t_{2}
\end{array}\right|=k_{i}^{2} A-k_{i} B+C=0
$$

with

$$
A=\operatorname{det}\left|\begin{array}{ll}
s_{1} & t_{1} \\
s_{2} & t_{2}
\end{array}\right|, \quad B=-\operatorname{det}\left|\begin{array}{ll}
r_{1} & t_{1} \\
r_{2} & t_{2}
\end{array}\right|, \quad C=\operatorname{det}\left|\begin{array}{ll}
r_{1} & s_{1} \\
r_{2} & s_{2}
\end{array}\right| .
$$

Interestingly enough, above coefficients (6.49) characterize the dual direction of $\overline{\mathcal{D}}^{v}$, i.e., a nonzero co-vector $\omega^{\prime}=A d p_{11}+B d p_{12}+C d p_{22} \in V^{*}$, defined up to a non-zero constant, such that

$$
\overline{\mathcal{D}}^{v}=\operatorname{ker} \omega^{\prime}
$$

This dual perspective on $\overline{\mathcal{D}}^{v}$ allows to rewrite (6.48) as

$$
\omega^{\prime}\left(k_{i}^{2} \partial_{p_{11}}-k_{i} \partial_{p_{12}}+\partial_{p_{22}}\right)=0
$$

In turn, (6.51) dictates the form of $\omega^{\prime}$ :

$$
\omega^{\prime}=A d p_{11}+B d p_{12}+\left(B k_{i}-A k_{i}^{2}\right) d p_{22}, \quad(A, B) \neq(0,0) .
$$

Together, (6.52) and (6.50) produce a simplified expression

$$
\overline{\mathcal{D}}^{v}=\left\langle\left(A k_{i}^{2}-B k_{i}\right) \partial_{p_{11}}+A \partial_{p_{22}},-B \partial_{p_{11}}+A \partial_{p_{12}}\right\rangle,
$$

which, in comparison with (6.45), depending on 6 parameters, needs only 2 of them, or even 1 , if a non-zero constant is neglected. Now, thanks to (6.53), it is easier to see that the space (6.47) is $1 \mathrm{D}$. Indeed, (6.47) reads

$$
\Omega\left(h_{i}, \overline{\mathcal{D}}^{v}\right)=\left\langle\left(A k_{i}^{2}-B k_{i}\right) \partial_{p_{1}}+A k_{i} \partial_{p_{2}},-B \partial_{p_{1}}+A \partial_{p_{2}}+A k_{i} \partial_{p_{1}}\right\rangle
$$


where the first vector equals the second multiplied by $k_{i}$, so that it can be further simplified:

$$
\Omega\left(h_{i}, \mathcal{D}^{v}\right)=\left\langle\left(A k_{i}-B\right) \partial_{p_{1}}+A \partial_{p_{2}}\right\rangle
$$

We can pass to the other condition dictated by (6.46). In particular, the subspace

$$
\begin{aligned}
\Omega\left(\bar{h}, \overline{\mathcal{D}}^{v}\right)=\langle & \left\langle a\left(-\left(k_{1}+k_{2}+k_{3}-k_{i}\right) \partial_{p_{1}}+\partial_{p_{2}}\right)+b \partial_{p_{1}},\right. \\
& \left.\left(-\left(k_{1} k_{2}+k_{1} k_{3}+k_{2} k_{3}\right) \partial_{p_{1}}+k_{i} \partial_{p_{2}}\right)+b\left(k_{i} \partial_{p_{1}}+\partial_{p_{2}}\right)\right\rangle \\
= & \left\langle\left(b-a\left(k_{1}+k_{2}+k_{3}-k_{i}\right)\right) \partial_{p_{1}}+a \partial_{p_{2}},\right. \\
& \left.\left(b k_{i}-a\left(k_{1} k_{2}+k_{1} k_{3}+k_{2} k_{3}\right)\right) \partial_{p_{1}}+\left(a k_{i}+b\right) \partial_{p_{2}}\right\rangle
\end{aligned}
$$

must be $1 \mathrm{D}$, i.e.,

$$
\operatorname{det}\left|\begin{array}{cc}
b-a\left(k_{1}+k_{2}+k_{3}-k_{i}\right) & a \\
b k_{i}-a\left(k_{1} k_{2}+k_{1} k_{3}+k_{2} k_{3}\right) & a k_{i}+b
\end{array}\right|=\prod_{j \neq i}\left(b-a k_{j}\right)=0 .
$$

Above equation (6.56), makes it evident that, for any $i=1,2,3$, the space (6.54) is $1 \mathrm{D}$ if and only if

$$
b=a k_{j}, \quad j \in\{1,2,3\} \backslash\{i\}, \quad a \neq 0,
$$

which corresponds to

$$
\bar{h}=D_{1}+k_{j} D_{2}, \quad j \in\{1,2,3\} \backslash\{i\} .
$$

Plugging (6.57) into (6.55), we find a unique vector

$$
\left(k_{i}+k_{j}-k_{1}-k_{2}-k_{3}\right) \partial_{p_{1}}+\partial_{p_{2}}
$$

generating $\Omega\left(\bar{h}, \mathcal{D}^{v}\right)$. Introducing the complement $c(i, j)$ of $\{i, j\}$ in $\{1,2,3\}$, (6.59) reads

$$
\Omega\left(\bar{h}, \mathcal{D}^{v}\right)=\left\langle-k_{c(i, j)} \partial_{p_{1}}+\partial_{p_{2}}\right\rangle, \quad j \in\{1,2,3\} \backslash\{i\} .
$$

To conclude the proof, recall that, besides their one-dimensionality, (6.46) also requires the equality of the subspaces (6.53) and (6.54), i.e., $\Omega\left(h_{i}, \overline{\mathcal{D}}^{v}\right)=\left\langle\left(A k_{i}-B\right) \partial_{p_{1}}+A \partial_{p_{2}},-k_{c(i, j)} \partial_{p_{1}}+\partial_{p_{2}}\right\rangle$ $=\Omega\left(\bar{h}, \mathcal{D}^{v}\right)$ or, alternatively,

$$
\operatorname{det}\left|\begin{array}{cc}
A k_{i}-B & A \\
-k_{c(i, j)} & 1
\end{array}\right|=0
$$

Thanks to $(6.60)$,

$$
B=A\left(k_{i}+k_{c(i, j)}\right), \quad j \in\{1,2,3\} \backslash\{i\},
$$

and (6.61) allows to eliminate $B$ from (6.53):

$$
\overline{\mathcal{D}}^{v}=\left\langle\left(A k_{i}^{2}-A\left(k_{i}+k_{c(i, j)}\right) k_{i}\right) \partial_{p_{11}}+A \partial_{p_{22}},-A\left(k_{i}+k_{c(i, j)}\right) \partial_{p_{11}}+A \partial_{p_{12}}\right\rangle .
$$

Being a non-zero constant ${ }^{12}, A$ can also be removed from (6.62), which becomes:

$$
\overline{\mathcal{D}}^{v}=\left\langle-k_{c(i, j)} k_{i} \partial_{p_{11}}+\partial_{p_{22}},\left(k_{c(i, j)}+k_{i}\right) \partial_{p_{11}}+\partial_{p_{12}}\right\rangle .
$$

\footnotetext{
${ }^{12}$ If $A=0$, then from (6.53) follows $\operatorname{dim} \bar{D}^{v}=1$.
} 
To enlighten the conclusions, it is useful to rewrite together (6.58) and (6.63) above: there are exactly 2 distributions $\overline{\mathcal{D}}$ which are "compatible" (in the sense of (6.46)) with the distribution $\mathcal{D}_{i}$ given by (6.39). More precisely, their horizontal and vertical parts are

$$
\bar{h}=D_{1}+k_{j} D_{2}, \quad \overline{\mathcal{D}}^{v}=\left\langle-k_{c(i, j)} k_{i} \partial_{p_{11}}+\partial_{p_{22}},\left(k_{c(i, j)}+k_{i}\right) \partial_{p_{11}}-\partial_{p_{12}}\right\rangle
$$

respectively, for the only two possible values of $j \in\{1,2,3\} \backslash\{i\}$. One only needs to realize that (6.64) is one of the $\mathcal{D}_{i}^{v}$ 's from (6.44). To this end, rewrite (6.44) replacing $i$ with $j$ : $\mathcal{D}_{j}^{v}=\left\langle-\left(k_{1}+k_{2}+k_{3}-k_{j}\right) \partial_{p_{11}}+\partial_{p_{12}},-\left(k_{1} k_{2}+k_{1} k_{3}+k_{2} k_{3}\right) \partial_{p_{11}}+k_{j} \partial_{p_{12}}-\partial_{p_{22}}\right\rangle$, and subtract from the second vector the first one multiplied by $k_{j}$ :

$$
\begin{aligned}
\mathcal{D}_{j}^{v}=\langle & -\left(k_{1}+k_{2}+k_{3}-k_{j}\right) \partial_{p_{11}} \\
& \left.+\partial_{p_{12}},\left(k_{j}\left(k_{1}+k_{2}+k_{3}-k_{j}\right)-\left(k_{1} k_{2}+k_{1} k_{3}+k_{2} k_{3}\right)\right) \partial_{p_{11}}+\partial_{p_{22}}\right\rangle .
\end{aligned}
$$

Formulas

$$
k_{j}\left(k_{1}+k_{2}+k_{3}-k_{j}\right)=k_{j} \sum_{l \neq j} k_{l}, \quad k_{1} k_{2}+k_{1} k_{3}+k_{2} k_{3}=k_{j} \sum_{l \neq j} k_{l}+\prod_{l \neq j} k_{l},
$$

show that the coefficient of $\partial_{p_{11}}$ in the second vector of (6.65) reduces to $-\prod_{l \neq j} k_{l}$. Hence, (6.65) reads

$$
\mathcal{D}_{j}^{v}=\left\langle-\left(\prod_{l \neq j} k_{l}\right) \partial_{p_{11}}+\partial_{p_{22}},\left(\sum_{l \neq j} k_{l}\right) \partial_{p_{11}}-\partial_{p_{12}}\right\rangle .
$$

Comparing now (6.64) with (6.66) it is evident that $\overline{\mathcal{D}}^{v}$ must equal $\mathcal{D}_{j}^{v}$, with $j \neq i$, and the proof of the "homogeneous" case is complete.

To deal with the general case, denote by $\mathcal{E}$ the equation determined by $F$ as in (6.41), with $c=0$, and by $\mathcal{E}_{c}$ a "inhomogeneous" equation, i.e., one with $c \neq 0$. Observe that there is a natural identification $i_{c}:\left\langle D_{1}, D_{2}\right\rangle \longrightarrow\left\langle D_{1}-c \partial_{p_{11}}, D_{2}\right\rangle$ between horizontal planes, giving rise to an automorphism $\varphi_{c}:=i_{c} \oplus \mathrm{id}_{V M^{(1)}}$ of $\mathcal{C}^{1}$. Easy computations show that $\varphi_{c}\left(\mathcal{D}_{i}\right), i=1,2,3$, are precisely the three distributions associated with the factors of the symbol of $\mathcal{E}_{c}$, and plainly $\Omega\left(h, \mathcal{D}^{v}\right)=\Omega\left(\varphi_{c}(h), \varphi_{c}\left(\mathcal{D}^{v}\right)\right)$. So, the "inhomogeneous" case reduces to the "homogeneous" one, which has been established above.

Corollary 6.17 (proof of statement 1 of Theorem 3.5). Let $\mathcal{E}=\{F=0\}$ be a quasi-linear $3^{\text {rd }}$ order $P D E$, and $U \subseteq M^{(1)}$ the open locus where the bundle $\mathcal{V}_{\mathrm{II}}^{\mathcal{E}}$ is not empty. Then $\mathcal{V}^{\mathcal{E}}=$ $\bigcup_{i=1}^{3} \mathbb{P} \mathcal{D}_{i}$ on $U$, where $\left.\mathcal{D}_{i} \subseteq \mathcal{C}\right|_{U}$ are orthogonal (see Definition 4.4 ) with respect to the metasymplectic structure on $M^{(1)}$ and $\mathcal{E}=\mathcal{E}_{\mathcal{D}_{i}}$ on $U$, for all $i=1,2,3$.

If $\mathcal{E}=\{F=0\}$ is a quasi-linear $3^{\text {rd }}$ order PDE, and $m^{1} \in M^{(1)}$ is such that $\left(\mathcal{V}_{\mathrm{II}}^{\mathcal{E}}\right)_{m_{1}} \neq \varnothing$, then it means that the function $\Delta$ (see Remark 5.6) is positive on the whole fibre $M_{m^{1}}^{(1)}$ and, hence, on the neighbouring fibres. So, $U$ is indeed open.

Proof of Corollary 6.17. Let $m^{1} \in U$. Then $\mathcal{V}_{\text {II }}^{\mathcal{E}} \neq \varnothing$, i.e., there is a point $m^{2} \in \mathcal{E}_{m^{1}}$ such that $\mathrm{Smbl}_{m^{2}} F$ is fully decomposable. But, in view of the quasi-linearity of $F$, this means that $\mathrm{Smbl} F$ is fully decomposable on the whole fibre $\mathcal{E}_{m^{1}}$, i.e., $\mathcal{E}$ fullfills the hypotheses of Lemma 6.16 on $U$. 


\subsubsection{Proof of the statement 2 of Theorem 3.5}

The first part of this statement merely rephrases the fist part of statement 1 .

Proving its second part consists in completing the proof of Lemma 6.8 above with the computation of $\mathcal{V}_{\mathrm{II}}^{\mathcal{E}}$, which corresponds to the quadratic factor (see Section 5.3) of (6.16). Formally, the procedure to get a description of $\mathcal{V}_{\text {II }}^{\mathcal{E}}$ out of the quadratic factor of (6.16) is the same as that for obtaining (6.17) from its linear factor, but it is more involved when it comes to computations, and this is the reason why we kept them separate.

So, suppose that $\mathcal{V}_{\mathrm{II}}^{\mathcal{E}} \neq \varnothing$. This means that there are points where the quadratic factor of (6.16) admits real roots. If $\Delta$ is the discriminant of such quadratic factor, then one its factor is given by

$$
\xi_{1}-\frac{T\left(p_{112}-f_{211}\right)-R\left(p_{222}-f_{222}\right)+\sqrt{\Delta}}{2\left(T\left(p_{122}-f_{212}\right)-S\left(p_{222}-f_{222}\right)\right)} \xi_{2},
$$

and the corresponding line $H_{1}$ is obtained by replacing ${ }^{13} \xi_{i}$ with $\left.D_{i}\right|_{\mathcal{E}}$ in (6.67). Now it is possible to assign certain values (namely $(0,0,0),(1,0,0),(0,1,0),(0,0,1))$ to the internal parameters of $\mathcal{E}$ (which are $p_{112}, p_{122}, p_{222}$ ), in such a way that the corresponding lines $H_{1}$ do not lie all in the same $3 \mathrm{D}$ subspace of $\mathcal{C}^{1}$. This shows that $\mathcal{V}_{\mathrm{II}}^{\mathcal{E}}$ cannot contain any linear irreducible component.

In order to prove that

$$
\mathcal{E}_{\mathcal{D}_{1}}=\mathcal{E}_{\mathcal{V}_{\text {II }}^{\mathcal{E}}}
$$

observe that the " $\supseteq$ " inclusion follows from the fact that, by definition, $\mathcal{V}_{\text {II }}^{\mathcal{E}}$ is made of characteristic lines of $\mathcal{E}_{\mathcal{D}_{1}}$ which are also strongly characteristic by Proposition 6.4, so that all Lagrangian planes containing a line in $\mathcal{V}_{\text {II }}^{\mathcal{E}}$ belong also to $\mathcal{E}_{\mathcal{D}_{1}}$ (see also Definition 5.2).

Inclusion " $\subseteq$ " can be obtained by a straightforward computation: indeed the $3 \times 5$ matrix whose first line is formed by the components of $H_{1}$ and the remaining ones by the components of the vectors spanning a generic Lagrangian plane belonging to $\mathcal{E}_{\mathcal{D}_{1}}$, is of rank 2 . The same result is attained by considering the line $H_{2}$ obtained by changing the sign of $\sqrt{\Delta}$ in (6.67).

\subsubsection{Proof of the statement 3 of Theorem 3.5}

Given any $3 \mathrm{D}$ sub-distribution $\mathcal{D} \subseteq \mathcal{C}^{1}$, and the corresponding Goursat-type equation $\mathcal{E}_{\mathcal{D}}$ (see (3.4)), we are now in position to clarify how many and which sort of elements can be found in the set

$$
\left\{\widetilde{\mathcal{D}} \mid \widetilde{\mathcal{D}} \text { is a } 3 \mathrm{D} \text { sub-distribution of } \mathcal{C}^{1} \text { such that } \mathcal{E}_{\widetilde{\mathcal{D}}}=\mathcal{E}_{\mathcal{D}}\right\}
$$

More precisely,

1) in the points of $M^{(1)}$ where $\operatorname{dim} \mathcal{D}^{v}=3$, we have $\mathcal{E}_{\mathcal{D}}=M^{(2)}$, so that the latter cannot be even considered as a $3^{\text {rd }}$ order PDE according to our understanding of PDEs (see Section 2.1), and this case must be excluded a prior $^{14}$;

2 ) in the points of $M^{(1)}$ where $\operatorname{dim} \mathcal{D}^{v}=1$, the equation $\mathcal{E}_{\mathcal{D}}$ is non-linear and the set (6.68) contains only $\mathcal{D}$;

3 ) in the points of $M^{(1)}$ where $\operatorname{dim} \mathcal{D}^{v}=2$, the equation $\mathcal{E}_{\mathcal{D}}$ is quasi-linear and the set (6.68) contains three elements $\mathcal{D}_{i}, i=1,2,3$, possibly repeated and comprising $\mathcal{D}$ itself, which are orthogonal each other (see Definition 4.4).

\footnotetext{
${ }^{13}$ This is a circumstance where the $\xi_{i}$ 's and the $D_{i}$ 's are vector fields, not vectors (still, this notation is consistent with the conventions established in Remark 3.6).

${ }^{14}$ Still, the description of the set (6.68) is very easy: it contains only $\mathcal{D}$, which in turn coincides with $V M^{(1)}$.
} 
Proof of 2 and 3. Let $\widetilde{\mathcal{D}}$ be an element of the set (6.68). Then, in particular,

$$
\mathcal{V}^{\mathcal{E}_{\mathcal{D}}}=\mathcal{V}^{\mathcal{E}_{\widetilde{\mathcal{D}}}}
$$

In view of Corollary 6.2, equality (6.69) implies

$$
\mathbb{P} \mathcal{D} \subseteq \mathcal{V}^{\mathcal{E}_{\tilde{\mathcal{D}}}}, \quad \mathbb{P} \widetilde{\mathcal{D}} \subseteq \mathcal{V}^{\mathcal{E}_{\mathcal{D}}}
$$

In other words, condition (3.5) is fulfilled by both equations $\mathcal{E}_{\widetilde{\mathcal{D}}}$ and $\mathcal{E}_{\mathcal{D}}$, so that the statements 1 and 2 of Theorem 3.5 (see above Sections 6.4.1 and 6.4.2) can be made use of. To this end, rewrite $(6.70)$ as

$$
\begin{aligned}
& \mathcal{V}^{\mathcal{E}_{\widetilde{\mathcal{D}}}}=\mathbb{P} \mathcal{D} \cup \mathcal{V}_{\mathrm{II}}^{\mathcal{E}_{\widetilde{\mathcal{D}}}}, \\
& \mathcal{V}^{\mathcal{E}_{D}}=\mathbb{P} \widetilde{\mathcal{D}} \cup \mathcal{V}_{\mathrm{II}}^{\mathcal{E}_{\mathcal{D}}},
\end{aligned}
$$

respectively. Because of statement 1 (reps., 2) of Theorem 3.5, from (6.71) it follows that $\mathcal{E}_{\widetilde{\mathcal{D}}}$ is quasi-linear (resp., non-linear) if and only if $\operatorname{dim} \mathcal{D}^{v}=2$ (resp., 1) and from (6.72) it follows that $\mathcal{E}_{\mathcal{D}}$ is quasi-linear (resp., non-linear) if and only if $\operatorname{dim} \widetilde{\mathcal{D}}^{v}=2$ (resp., 1). Summing up, $\operatorname{dim} \mathcal{D}^{v}=\operatorname{dim} \widetilde{\mathcal{D}}^{v}=1,2$, and the two cases can be treated separately.

Directly from (6.71) and (6.72) we get

$$
\mathbb{P} \widetilde{\mathcal{D}} \cup \mathcal{V}_{\mathrm{II}}^{\mathcal{E}_{\widetilde{\mathcal{D}}}}=\mathbb{P} \mathcal{D} \cup \mathcal{V}_{\mathrm{II}}^{\mathcal{E}_{\widetilde{\mathcal{D}}}}, \quad \mathbb{P} \mathcal{D} \cup \mathcal{V}_{\mathrm{II}}^{\mathcal{E}_{\mathcal{D}}}=\mathbb{P} \widetilde{\mathcal{D}} \cup \mathcal{V}_{\mathrm{II}}^{\mathcal{E}_{\mathcal{D}}}
$$

Prove now 2. If $\operatorname{dim} \mathcal{D}^{v}=1$, then also $\operatorname{dim} \widetilde{\mathcal{D}}^{v}=1$ and the statement 2 of Theorem 3.5 (see Section 6.4.2) guarantees that $\mathcal{V}_{\text {II }}^{\mathcal{E}_{\widetilde{\mathcal{D}}}}$ (resp., $\mathcal{V}_{\mathrm{II}}^{\mathcal{E}_{\mathcal{D}}}$ ) cannot contain $\mathbb{P \mathcal { D }}$ (resp., $\mathbb{P} \widetilde{\mathcal{D}}$ ). It follows from (6.73) that $\mathbb{P} \widetilde{\mathcal{D}}=\mathbb{P} \mathcal{D}$.

Finally prove 3 . If $\operatorname{dim} \mathcal{D}^{v}=2$, then also $\operatorname{dim} \widetilde{\mathcal{D}}^{v}=2$ and, in view of the statement 1 of Theorem 3.5 (see Section 6.4.1), $\mathcal{V}_{\mathrm{II}}^{\mathcal{E}_{\widetilde{\mathcal{D}}}}$ (resp., $\mathcal{V}_{\mathrm{II}}^{\mathcal{E}_{\mathcal{D}}}$ ) is either empty, in which case $\mathbb{P} \widetilde{\mathcal{D}}=\mathbb{P} \mathcal{D}$, or consists of two, possibly repeated, distributions "orthogonal" to $\widetilde{\mathcal{D}}$ (resp., $\mathcal{D}$ ).

The result presented in this section mirrors the analogous result for classical multi-dimensional MAEs (see [2, Theorem 1]), but displays some new and unexpected features: the threefold multiplicity of the notion of orthogonality and the distinction of the cases according to the dimension of the vertical part.

\section{$7 \quad$ Intermediate integrals of Goursat-type $3^{\text {rd }}$ order MAEs}

Theorem 3.5 established a link between $3^{\text {rd }}$ order MAEs of Goursat-type, i.e., non-linear PDEs of order three, and 3D sub-distributions $\mathcal{D}$ of $\mathcal{C}^{1}$, i.e., linear objects involving (at most) secondorder partial derivatives. Besides its aesthetic value, such a perspective also allows to formulate concrete results concerning the existence of solutions, as the first integrals of $\mathcal{D}$ can be made use of in order to find intermediate integrals of $\mathcal{E}_{\mathcal{D}}$, along the same lines of the classical case (see [2, Section 6.3] and [3], where a more general concept of intermediate integral is exploited to explicitly construct solutions to $2^{\text {nd }}$ order parabolic MAEs). Proposition 7.4 is the main result of this last section, showing that, for a Goursat-type $3^{\text {rd }}$ order MAE $\mathcal{E}$, the notions of an intermediate integral of $\mathcal{E}$ and of a first integral of any distribution $\mathcal{D}$ such that $\mathcal{E}_{\mathcal{D}}=\mathcal{E}$ are actually the same. To facilitate its proof, we deemed it convenient to introduce equations of the form $\mathcal{E}=\mathcal{E}_{\mathcal{V}}$ where $\mathcal{V}=\mathbb{P} \check{\mathcal{D}}$, with, for the first time in this paper, $\operatorname{dim} \check{\mathcal{D}}=2$. By analogy with (3.4), we still write $\mathcal{E}_{\check{\mathcal{D}}}$ instead of $\mathcal{E}_{\mathbb{P} \check{\mathcal{D}}}$, but we warn the reader that, unlike all the cases considered so far, $\mathcal{E}_{\breve{\mathcal{D}}}$ is actually a system of two independent equations (see Lemma 7.2 below). 
Recall that a function $f \in C^{\infty}\left(M^{(1)}\right)$ determines, in the neighborhood of an its non-singular point, the hyperplane distribution $\operatorname{ker} d f$ on $M^{(1)}$, each of whose leaves is identified by a value $c \in \mathbb{R}$. Following the same notation as [2], we set

$$
\left(M^{(1)}\right)_{f-c}:=\left\{m^{1} \in M^{(1)} \mid f\left(m^{1}\right)=c\right\} .
$$

Definition 7.1. A function $f \in C^{\infty}\left(M^{(1)}\right)$ is an intermediate integral of a PDE $\mathcal{E} \subseteq M^{(2)}$ if

$$
\left(M^{(1)}\right)_{f-c}^{(1)} \subseteq \mathcal{E}
$$

for all $c \in \mathbb{R}$.

Inclusion (7.1) can be interpreted as follows: if $f=c$ defines a $2^{\text {nd }}$ order PDE (in the sense given in Section 2.1), then any solution of such an equation is also a solution of the $3^{\text {rd }}$ order equation $\mathcal{E}$.

Lemma 7.2. Let $\check{\mathcal{D}} \subset \mathcal{C}^{1}$ be a $2 D$ non-vertical sub-distribution. Then $\mathcal{E}_{\breve{\mathcal{D}}} \longrightarrow M^{(1)}$ is a nonlinear bundle of rank 2 .

Proof. To simplify the notations, let $\mathcal{E}:=\mathcal{E}_{\mathcal{D}^{\circ}}$. Then, fix $m^{1} \in M^{(1)}$ and observe that $\mathcal{E}_{m^{1}}=$ $\left\{m^{2} \in M^{(2)} \mid L_{m^{2}} \cap \check{\mathcal{D}}_{m^{1}} \neq 0\right\}$ is a $2 \mathrm{D}$ manifold. Indeed, $\mathcal{E}_{m^{1}}$ is ${ }^{15}$ a rank-one bundle over the $1 \mathrm{D}$ manifold $\mathbb{P} \check{\mathcal{D}}$, its fibres being the prolongations of the lines lying in $\check{\mathcal{D}}$ (see [8, Proposition 2.5]).

To prove non-linearity of $\mathcal{E}$, write down $\check{\mathcal{D}}$ in local coordinates as

$$
\check{\mathcal{D}}=\left\langle a_{i} D_{1}+b_{i} D_{2}+R_{i} \partial_{p_{11}}+S_{i} \partial_{p_{12}}+T_{i} \partial_{p_{22}} \mid i=1,2\right\rangle
$$

and observe that $\mathcal{E}$ is given by the vanishing of the five $4 \times 4$ minors of the matrix

$$
\left|\begin{array}{ccccc}
a_{1} & b_{1} & R_{1} & S_{1} & T_{1} \\
a_{2} & b_{2} & R_{2} & S_{2} & T_{2} \\
1 & 0 & p_{111} & p_{112} & p_{122} \\
0 & 1 & p_{112} & p_{122} & p_{222}
\end{array}\right| .
$$

Tedious computations show that the only cases when such minors are quasi-linear, is that either when $\check{\mathcal{D}}$ fails to be two-dimensional or when $\check{\mathcal{D}}$ is vertical, which are forbidden by the hypotheses.

Corollary 7.3. Let $\check{\mathcal{D}} \subset \mathcal{C}^{1}$ be a $2 D$ sub-distribution contained in ker $d f$. Then for any $m^{1} \in M^{(1)}$ there is a $m^{2} \in M_{m^{1}}^{(2)}$ such that $L_{m^{2}} \subset \operatorname{ker} d_{m^{1}} f$ and $L_{m^{2}} \cap \check{\mathcal{D}}_{m^{1}}=0$.

Proof. Assume for contradiction that

$$
\left\{m^{2} \in M^{(2)} \mid L_{m^{2}} \subset \operatorname{ker} d_{m^{1}} f\right\} \subseteq\left\{m^{2} \in M^{(2)} \mid L_{m^{2}} \cap \check{\mathcal{D}}_{m^{1}} \neq 0\right\}
$$

and set $c:=f\left(m^{1}\right)$. Then (7.2) means that, over the point $m^{1}$,

$$
\left(M^{(1)}\right)_{f-c}^{(1)} \subseteq \mathcal{E}_{\check{\mathcal{D}}},
$$

which is impossible, since the left-hand side of (7.3) is linear while its right-hand side is either empty (in the case when $\check{\mathcal{D}} \subseteq V M^{(1)}$ ) or non-linear, thanks to above Lemma 7.2.

Proposition 7.4. Let $\mathcal{E}$ be a Goursat-type $3^{\text {rd }}$ order $M A E$ and $f \in C^{\infty}\left(M^{(1)}\right)$. Then the following statements are equivalent:

\footnotetext{
${ }^{15}$ This step may require the restriction to an open and dense subset.
} 
- $f$ is an intermediate integral of $\mathcal{E}$;

- there exists a $3 D$ distribution $\mathcal{D} \subset \mathcal{C}^{1}$ such that $f$ is a first integral of $\mathcal{D}$ and $\mathcal{E}_{\mathcal{D}}=\mathcal{E}$.

Proof. If $f$ is a first integral of $\mathcal{D}$, then

$$
\mathcal{D} \subseteq \mathcal{C}^{1} \cap \operatorname{ker} d f \subseteq \mathcal{C}^{1}
$$

is a $(3,4,5)$-type flag of distributions $\left(\mathcal{C}^{1}\right.$ does not posses first integrals). By definition,

$$
\left(M^{(1)}\right)_{f-c}^{(1)}=\left\{m^{2} \in M^{(2)}\left|f\left(\pi_{2,1}\left(m^{2}\right)\right)=c, d f\right|_{L_{m^{2}}}=0\right\}
$$

and condition $\left.d f\right|_{L_{m^{2}}}=0$ means precisely that the $2 \mathrm{D}$ subspace $L_{m^{2}}$ of $\mathcal{C}^{1}$ is also contained in ker $d f$, i.e., $L_{m^{2}}$ lies in the $4 \mathrm{D}$ subspace $\mathcal{C}^{1} \cap \operatorname{ker} d f$ of $\mathcal{C}^{1}$. Because of (7.4), the 3D subspace $\mathcal{D}$ is also contained in $\mathcal{C}^{1} \cap \operatorname{ker} d f$ and, as such, it cannot fail to non-trivially intersect $L_{m^{2}}$. This means that $m^{2} \in \mathcal{E}_{\mathcal{D}}$, and (7.1) follows from the arbitrariness of $m^{2}$.

Suppose, conversely, that $f$ is an intermediate integral of $\mathcal{E}$. In view of (7.5) and inclusion (7.1), one has that

$$
L_{m^{2}} \subseteq \operatorname{ker} d f \Rightarrow L_{m^{2}} \cap \mathcal{D}_{m^{1}} \neq 0
$$

for any distribution $\mathcal{D}$ such that $\mathcal{E}_{\mathcal{D}}=\mathcal{E}$. We wish to show that, there is at least one among these distributions such that $f$ is an its first integral, i.e., that the first inclusion of (7.4) is satisfied. Towards a contradiction, suppose that no distribution $\mathcal{D}$ is contained in ker $d f$, i.e., that $\check{\mathcal{D}}_{f}:=\mathcal{D} \cap$ ker $d f$ is a $2 \mathrm{D}$ sub-distribution of the $4 \mathrm{D}$ distribution $\mathcal{C}^{1} \cap$ ker $d f$, for all distributions $\mathcal{D}$ such that $\mathcal{E}_{\mathcal{D}}=\mathcal{E}$. Then, by Corollary 7.3 , it is possible to find a (2D subspace) $L_{m^{2}}$ which is contained in $\mathcal{C}^{1} \cap$ ker $d f$ and also trivially intersects one $\check{\mathcal{D}}_{f}$, thus violating (7.6).

Corollary 7.5. If the derived flag of $\mathcal{D}$ never reaches $T M^{(1)}$, then $\mathcal{E}_{\mathcal{D}}$ admits an intermediate integral.

\section{Acknowledgments}

The authors wish to express their gratitude towards the anonymous referees whose comments contributed to shape the paper into its final form. The authors thank C. Ciliberto, E. Ferapontov and F. Russo for stimulating discussions. The research of the first author has been partially supported by the project "Finanziamento giovani studiosi - Metriche proiettivamente equivalenti, equazioni di Monge-Ampère e sistemi integrabili", University of Padova 2013-2015, by the project "FIR (Futuro in Ricerca) 2013 - Geometria delle equazioni differenziali". The research of the second author has been partially supported by the Marie Skłodowska-Curie Action No 654721 "GEOGRAL", by the University of Salerno, and by the project P201/12/G028 of the Czech Republic Grant Agency (GA ČR). Both the authors are members of G.N.S.A.G.A. of I.N.d.A.M.

\section{References}

[1] Agafonov S.I., Ferapontov E.V., Systems of conservation laws in the setting of the projective theory of congruences: reducible and linearly degenerate systems, Differential Geom. Appl. 17 (2002), 153-173.

[2] Alekseevsky D.V., Alonso Blanco R., Manno G., Pugliese F., Contact geometry of multidimensional MongeAmpère equations: characteristics, intermediate integrals and solutions, Ann. Inst. Fourier (Grenoble) 62 (2012), 497-524, arXiv:1003.5177.

[3] Alekseevsky D.V., Alonso Blanco R., Manno G., Pugliese F., Finding solutions of parabolic Monge-Ampère equations by using the geometry of sections of the contact distribution, Differential Geom. Appl. 33 (2014), suppl., 144-161. 
[4] Alonso Blanco R., Manno G., Pugliese F., Contact relative differential invariants for non generic parabolic Monge-Ampère equations, Acta Appl. Math. 101 (2008), 5-19.

[5] Alonso Blanco R., Manno G., Pugliese F., Normal forms for Lagrangian distributions on 5-dimensional contact manifolds, Differential Geom. Appl. 27 (2009), 212-229, arXiv:0707.0683.

[6] Bächtold M.J., Fold-type solution singularities and charachteristic varieties of non-linear PDEs, Ph.D. Thesis, Universität Zürich, 2009.

[7] Bächtold M.J., Moreno G., Remarks on non-maximal integral elements of the Cartan plane in jet spaces, J. Geom. Phys. 85 (2014), 185-195, arXiv:1208.5880.

[8] Bocharov A.V., Chetverikov V.N., Duzhin S.V., Khor'kova N.G., Krasil'shchik I.S., Samokhin A.V., Torkhov Yu.N., Verbovetsky A.M., Vinogradov A.M., Symmetries and conservation laws for differential equations of mathematical physics, Translations of Mathematical Monographs, Vol. 182, Amer. Math. Soc., Providence, RI, 1999.

[9] Boillat G., Sur l'équation générale de Monge-Ampère à plusieurs variables, C. R. Acad. Sci. Paris Sér. I Math. 313 (1991), 805-808.

[10] Boillat G., Sur l'équation générale de Monge-Ampère d'ordre supérieur, C. R. Acad. Sci. Paris Sér. I Math. 315 (1992), 1211-1214.

[11] Bryant R.L., Chern S.S., Gardner R.B., Goldschmidt H.L., Griffiths P.A., Exterior differential systems, Mathematical Sciences Research Institute Publications, Vol. 18, Springer-Verlag, New York, 1991.

[12] De Paris A., Vinogradov A.M., Scalar differential invariants of symplectic Monge-Ampère equations, Cent. Eur. J. Math. 9 (2011), 731-751, arXiv:1102.0426.

[13] De Poi P., Mezzetti E., Congruences of lines in $\mathbb{P}^{5}$, quadratic normality, and completely exceptional MongeAmpère equations, Geom. Dedicata 131 (2008), 213-230, arXiv:0710.5110.

[14] Dubrovin B., Geometry of 2D topological field theories, in Integrable Systems and Quantum Groups (Montecatini Terme, 1993), Lecture Notes in Math., Vol. 1620, Springer, Berlin, 1996, 120-348, hep-th/9407018.

[15] Ferapontov E.V., Decomposition of higher-order equations of Monge-Ampère type, Lett. Math. Phys. 62 (2002), 193-198, nlin.SI/0205056.

[16] Goursat E., Sur les équations du second ordre à $n$ variables analogues à l'équation de Monge-Ampère, Bull. Soc. Math. France 27 (1899), 1-34.

[17] Kolář I., Michor P.W., Slovák J., Natural operations in differential geometry, Springer-Verlag, Berlin, 1993.

[18] Krasil'shchik J., Verbovetsky A., Geometry of jet spaces and integrable systems, J. Geom. Phys. 61 (2011), 1633-1674, arXiv:1002.0077.

[19] Kruglikov B., Lychagin V., Geometry of differential equations, in Handbook of Global Analysis, Elsevier Sci. B. V., Amsterdam, 2008, 725-771.

[20] Kushner A., Lychagin V., Rubtsov V., Contact geometry and non-linear differential equations, Encyclopedia of Mathematics and its Applications, Vol. 101, Cambridge University Press, Cambridge, 2007.

[21] Lax P.D., Milgram A.N., Parabolic equations, in Contributions to the Theory of Partial Differential Equations, Annals of Mathematics Studies, Vol. 33, Princeton University Press, Princeton, N. J., 1954, 167-190.

[22] Lychagin V.V., Singularities of multivalued solutions of nonlinear differential equations, and nonlinear phenomena, Acta Appl. Math. 3 (1985), 135-173.

[23] Lychagin V.V., Geometric theory of singularities of solutions of nonlinear differential equations, J. Sov. Math. 51 (1990), 2735-2757.

[24] Moreno G., Submanifolds in the Grassmannian of $n$-dimensional subspaces determined by a submanifold in the Grassmannian of $l$-dimensional subspaces, MathOverflow, 2013, available at http://mathoverflow . net/q/138544.

[25] Moreno G., The geometry of the space of Cauchy data of nonlinear PDEs, Cent. Eur. J. Math. 11 (2013), 1960-1981, arXiv:1207.6290.

[26] Morimoto T., Monge-Ampère equations viewed from contact geometry, in Symplectic Singularities and Geometry of Gauge Fields (Warsaw, 1995), Banach Center Publ., Vol. 39, Polish Acad. Sci., Warsaw, 1997, 105-121.

[27] Piccione P., Tausk D.V., The single-leaf Frobenius theorem with applications, Resenhas 6 (2005), 337-381, math.DG/0510555.

[28] Strachan I.A.B., On the integrability of a third-order Monge-Ampère type equation, Phys. Lett. A 210 (1996), 267-272.

[29] Vitagliano L., Characteristics, bicharacteristics and geometric singularities of solutions of PDEs, Int. J. Geom. Methods Mod. Phys. 11 (2014), 1460039, 35 pages, arXiv:1311.3477. 